\title{
Violence Domestique dans les \\ Communautés d'Immigrants: Études de Cas
}





\section{VIOLENCE DOMESTIQUE DANS LES COMMUNAUTÉS D'IMMIGRANTS: ÉTUDES DE CAS}

FERZANA CHAZE, BETHANY OSBORNE, ARCHANA MEDHEKAR, ET PURNIMA GEORGE

SERAPHINA SEURATAN, JASPREET KAUR, DENISE DEJONG, TERRI NEUFELD, MARIA AOSAF DAWD, KRUTTIKA NENE, ET KATRINA CHAHAL 


\section{() (1) $\odot \odot$}

Violence Domestique dans les Communautés d'Immigrants: Études de Cas de Ferzana Chaze, Bethany Osborne, Purnima George and Archana Medhekar est sous une licence Licence Creative Commons Attribution - Pas d'utilisation commerciale - Pas de modification 4.0 International, sauf indication contraire. 


\section{TABLE DES MATIĖRES}

Introduction 1

Méthodologie 2

Partie I. Les études de cas

1. Lecture des études de cas 7

2. Étude de cas 1: Ramandeep et Aman 8

3. Étude de cas 2: Zakia et Wasim 12

4. Étude de cas 3: Miremba et lames 16

5. Étude de cas 4: Sonali et Ravi 19

6. Étude de cas 5: Gagandeep et Kuldip 23

7. Étude de cas 6: Namrata et Dinesh 26

8. Étude de cas 7: Deepa et Amol 30

9. Étude de cas 8: Claudia et Henry 34

10. Étude de cas 9: Rabia et Ali 37

11. Étude de cas 10 : Anjana et Marc 40

12. Étude de cas 11: Shova et Anant 43

13. Étude de cas 12 : Bina et Udeep 47

14. Étude de cas 13: Elham et Dawood 50

15. Étude de cas 14 : Ritu et Satinder 54

16. Étude de cas 15: Ayesha et Kabir 57 
Partie II. Questions pour engager une réflexion et une discussion

17. Vulnérabilités intersectionnelles 63

18. Subjectivité et localisation sociale des praticiens 65

19. Politique et droit sociaux 66

20. Migration et transnationalisme 67

21. Soutien aux victimes de la violence domestique 68

22. Recherche 69 


\section{INTRODUCTION}

La violence domestique est un problème qui touche toutes les communautés. Dans ce livre, nous avons voulu retracer les expériences vécues par des femmes migrantes qui ont fait appel aux services sociaux et à l'appareil judiciaire pour assurer leur sécurité et celle de leurs enfants. Cet ouvrage vise à approfondir la compréhension des obstacles et des défis auxquels ces femmes immigrées ont été confrontées. Dans les pages suivantes, vous trouverez des informations sur la méthodologie de cette recherche, une brève recommandation des auteurs pour lire les études de cas, les 15 études de cas et des questions pour réfléchir et engager la discussion.

Ce livre est extrait de l'ouvrage «Violence domestique dans les communautés d'immigrants : études de cas » qui présente des affaires de femmes migrantes victimes de violence familiale à des fins pédagogiques. Le contenu de l'ouvrage original a été élaboré à partir de l’analyse des dossiers des affaires judiciaires classées de 15 femmes immigrées victimes de violence conjugale et vivant en Ontario. Les études de cas approfondies, qui ressortent de cette recherche, décrivent dans toute leur complexité les violences domestiques subies par ces femmes immigrées. Elles soulignent la vulnérabilité singulière des victimes, à la croisée des questions de race, de sexe et d'immigration. Le livre met également en lumière les différents dispositifs juridiques auxquels sont confrontées ces femmes et les défis qu'elles doivent relever pour reconstruire leur vie et celles de leurs enfants. Ce livre pose aussi un certain nombre de questions pour nourrir la réflexion des lecteurs, décrit les principales procédures judiciaires utilisées dans les affaires de violence conjugale et fournit un glossaire des termes utilisés dans les « études de cas ». Ce livre sera certainement une ressource précieuse pour les professionnels du secteur social et du droit, notamment les futurs travailleurs sociaux et techniciens en travail social. Il leur permettra de mieux comprendre la complexité des cas de violence domestique dans les familles de migrants et de développer des stratégies d'intervention adaptées à ces cultures. Pour consulter l'ouvrage original, veuillez cliquer sur ce lien. 


\section{MÉTHODOLOGIE}

Les cas présentés dans ce livre sont extraits de dossiers juridiques traités par le cabinet d'avocats Archana Medhekar. Ils retracent les expériences vécues par des femmes immigrantes, racialisées et victimes de violence domestique qui ont demandé une aide juridique au Canada. L'autorisation de mener cette recherche a été accordée par le Comité d’éthique de la recherche de l'Université Ryerson et le Research Ethics Board du Sheridan College en juin 2019. Toutes les affaires décrites dans cette recherche ont eu lieu au cours des dix dernières années. Elles ont été classées au moins un an avant le début de cette recherche. Il s'est donc écoulé un temps suffisant entre le classement de ces affaires et la publication de ce livre.

Toutes les précautions ont été prises pour qu’aucune femme ne se sente obligée de participer à cette étude et pour qu'elles y consentent de manière libre et éclairé. Le cabinet d'avocats d'Archana Medhekar a publié sur son site internet une affiche appelant des volontaires à participer à ce projet. Les anciennes clientes qui souhaitaient participer à cette recherche, et dont les cas répondaient aux critères de recevabilité, ont été invitées à entrer directement en contact avec Purnima George, l'une des chercheuses de l'étude. Après ce premier contact, Purnima a organisé une réunion téléphonique avec les volontaires pour leur décrire l'étude, répondre à leurs questions, informer les participantes qu'elles avaient le droit de se retirer de l'étude à tout moment et obtenir leur consentement éclairé. Le fait que les personnes intéressées se soient adressées à une seule chercheuse a permis à l'équipe de limiter toute influence involontaire sur la décision de ces femmes de participer à l'étude et de minimiser le nombre de personnes susceptibles de connaître l'identité des participantes, afin de garantir une meilleure confidentialité.

L'équipe de recherche a pris d'autres mesures pour s'assurer que l'identité des participantes demeurerait confidentielle. Une fois le consentement éclairé obtenu, l'équipe de recherche a créé des études de cas à partir des histoires vécues par ces femmes, principalement sur la base des copies des plaidoiries auprès de la Cour de la famille. Afin de mieux expliquer la complexité de chaque cas, la recherche a fait référence aux documents suivants : documents de la Cour de la famille (réponses, répliques, affidavits tels que : affidavits à l'appui de la garde ou du droit de visite, affidavits à l’appui des motions, mémoires tels que : mémoire de la conférence relative à la cause, mémoires de la conférence en vue d'une transaction, mémoires de la conférence de gestion de procès, ordonnances de la Cour : ordonnances et décisions écrites du juge); dossier de la Cour pénale : (dossiers de police tels que les copies de dénonciation, les transcriptions des appels au 911); documents de l'immigration: (copies des demandes auprès de la Commission de l'immigration et du statut de réfugié et les ordonnances de celle-ci); et autres documents : dossiers médicaux, rapports des droits de visite, rapports d'évaluation des besoins des enfants (L.R.O. chap. 12 par. 30) et rapports du bureau de l'avocat des enfants 
(BAE). Il a été décidé de créer des pseudonymes pour désigner les participantes à létude afin de permettre au lecteur de suivre plus facilement le récit. Une fois ce travail terminé, les études de cas ont été présentées aux participantes respectives pour qu'elles puissent vérifier les faits et incorporer leurs commentaires dans la version finale du document. Cette vérification a permis aux participantes d'examiner et d'approuver la façon dont elles étaient représentées dans l'étude de cas. L'équipe de recherche a aussi fourni aux participantes des informations sur des lignes d'assistance téléphonique d'urgence accessibles, au cas où elles se sentiraient traumatisées après avoir lu leur étude de cas. 



\section{PARTIE I LES ÉTUDES DE CAS}

La section précédente détaillait les méthodes utilisées pour créer les études de cas, qui constituent la partie essentielle de ce livre. La section suivante comprend les études de cas. Chaque étude commence par un bref profil socio-démographique des femmes et de leurs partenaires. L'étude décrit le parcours du couple avant la migration, les expériences vécues lors de l'installation, les violences domestiques et le règlement final de l'affaire.

Toutes les informations permettant d'identifier les personnes ont été modifiées (nom, ville d'origine, lieu de résidence), mais certains détails ont été conservés pour mieux appréhender certaines histoires. Des détails comme la situation au regard de l'immigration, les domaines d'activité professionnelle de chaque conjoint, le nombre et l'âge des enfants, la présence ou l'absence de membres de la famille et le bagage culturel sont utiles pour comprendre les circonstances de chacune de ces affaires. 



\section{1.}

\section{LECTURE DES ÉTUDES DE CAS}

Ces études sont basées sur des cas réels. Notre équipe de recherche a appris une chose en réalisant ce projet: tous ces cas basés sur des situations réelles ne laissent personne indifférent. Nous sommes toutes des professionnelles aguerries qui avons exercé diverses fonctions au sein des services sociaux et de la justice pénale, mais nous avons dû à certains moments de ce projet faire une pause et reconnaitre qu'il était difficile de lire les récits des violences subies par un si grand nombre de femmes. Nous conseillons donc aux lecteurs et aux lectrices, qui commencent à lire ces études, de prendre le temps nécessaire pour les assimiler. Lisez un ou deux cas à la fois et posez-vous les questions incluses dans ce livre pour réfléchir à ces affaires. Chacune de ces histoires témoigne d'une expérience violente dont la fin n’a pas toujours été «heureuse ». Elles ont connu un dénouement heureux quand ces femmes ont reçu un soutien et qu'elles ont pu aller de l'avant. C'est pourquoi ces femmes ont accepté de témoigner : elles souhaitaient que leurs expériences puissent aider d'autres femmes. C'est là l'objet principal de cette étude : favoriser l'apprentissage. Plus important encore, que peut nous apprendre l'étude de chacun de ces cas? Dans quelle mesure, cet acquis influera-t-il sur nos pratiques et nos services d'aide et nous permettra de répondre aux attentes de toutes ces femmes qui ont besoin d'aide?

Si vous vous sentez personnellement concerné par l'un de ces cas en raison d'une expérience personnelle ou professionnelle passée, nous vous recommandons de demander de l'aide. La violence est un problème qui nous touche tous : elle est omniprésente dans le monde qui nous entoure et, malheureusement, elle joue souvent un rôle important dans nos vies. Elle nous isole et nous enferme dans la solitude. Toutefois, la violence perd de sa force lorsque les gens se décident à demander de l'aide et à raconter leurs histoires. Lorsque nous parlons avec quelqu'un des effets de la violence sur notre personne, nous commençons à nous sentir moins seuls. 


\section{ÉTUDE DE CAS 1 : RAMANDEEP ET AMAN}

\begin{tabular}{|c|c|c|}
\hline Profil & Femme & Homme \\
\hline Nom & Ramandeep & Aman \\
\hline Âge au moment du mariage & 22 & 24 \\
\hline Âge* & 39 & 41 \\
\hline Pays d'origine & Inde & Inde \\
\hline Religion & Sikh & Sikh \\
\hline Éducation & Aucune information disponible & Aucune information disponible \\
\hline Connaissance de l'anglais & Compétences limitées & Compétences limitées \\
\hline Emploi avant la migration & Jamais travaillé & Aucune information disponible \\
\hline Emploi & $\begin{array}{l}\text { Travail à temps partiel en tant que } \\
\text { chauffeur de bus scolaire }\end{array}$ & $\begin{array}{l}\text { Employé à temps plein depuis } \\
\text { octobre } 1993\end{array}$ \\
\hline Catégorie d'immigration & $\begin{array}{l}\text { Ramandeep a immigré au Canada } \\
\text { avec sa famille avant son mariage. }\end{array}$ & $\begin{array}{l}\text { Regroupement familial : Ramandeep } \\
\text { a parrainé Aman dans le cadre du } \\
\text { programme de parrainage des } \\
\text { conjoints }\end{array}$ \\
\hline Statut d'immigrant* & Citoyen canadien & Aucune information disponible \\
\hline \multicolumn{3}{|c|}{$\begin{array}{l}\text { Nombre d'années de mariage* }: \mathbf{1 7} \\
\text { Enfants* : } \\
\text { - Fille : Guneet ( } 14 \text { ans ; troubles du comportement) } \\
\text { - Fils : Navdeep ( } 11 \text { ans ; diagnostiqué avec un TDAH et un trouble d'apprentissage) }\end{array}$} \\
\hline
\end{tabular}

*Au moment de la demande auprès de la Cour de la famille

\section{Parcours avant la migration}

La cérémonie de mariage a eu lieu en Inde : Ramandeep avait vingt-deux ans et Aman vingt-quatre ans. Le couple souhaitait vivre au Canada et Ramandeep a parrainé l'immigration d'Aman dans le cadre du programme 
de parrainage des conjoints. Six mois après le mariage, Aman est arrivé au Canada. On dispose de peu d'informations sur les antécédents du couple avant la migration, si ce n'est que leur mariage a été arrangé par leurs familles respectives.

\section{Installation au Canada}

Lorsqu'Aman est arrivé au Canada, le couple a vécu chez le père de Ramandeep pour économiser l'argent nécessaire à l'acquisition de leur première maison. Tout de suite après l'arrivée d'Aman, Ramandeep a compris que la vie avec son mari allait être difficile. Dès la première semaine, celui-ci a commencé à boire quotidiennement et à harceler Ramandeep et ses voisins pour qu'ils le conduisent en ville pour acheter de l'alcool. Aman a trouvé un travail dans le mois suivant son arrivée au Canada. Au début de leur mariage, Ramandeep travaillait à l'extérieur, mais quelques mois plus tard, elle a dû interrompre cette activité à la suite d'un accident. Aman a alors fait pression sur Ramandeep pour qu'ils fondent rapidement une famille et il a commencé à l'agresser verbalement en lui reprochant de ne pas avoir un enfant assez rapidement. Trois années après son arrivée au Canada, Aman a été condamné pour conduite en état alcoolique et son permis de conduire lui a été retiré pendant quelque temps. La consommation d'alcool et les violences verbales d'Aman n’ont pas cessé durant tout leur mariage.

Au bout de quatre ans de mariage, le couple a eu son premier enfant : une fille. Les violences psychologiques et verbales subies par Ramandeep se sont transformées en violences physiques. Lorsqu'il buvait, Aman battait régulièrement Ramandeep et la forçait à avoir des relations sexuelles avec lui. Espérant pouvoir sauver son mariage, Ramandeep ne parlait de ces violences à personne. Le couple a rapidement acheté une petite maison avec l'aide financière du père de Ramandeep. Ramandeep a commencé à dormir séparément et à vivre avec son bébé au sous-sol de la maison, en s'occupant des tâches ménagères et de l'éducation de son enfant. Mais Aman exigeait quotidiennement des rapports sexuels et la battait si elle ne s'y conformait pas. Lorsque sa fille a eu quatre ans, Ramandeep est tombée de nouveau enceinte d'un garçon. Aman a alors fait pression sur Ramandeep pour qu'elle avorte, mais celle-ci a refusé. Son nouveau-né présentait plusieurs problèmes médicaux à la naissance. Ramandeep a commencé à travailler les fins de semaine pour soutenir financièrement la famille grandissante. Dès le début du mariage, les ressources financières n'étaient pas réparties également au sein du couple. Ramandeep devait assumer seule les dépenses supplémentaires pour les enfants, notamment les dépenses médicales relatives aux soins de leur fils, alors qu'Aman envoyait de l'argent à ses frères en Inde pour entretenir sa propriété.

\section{Violence domestique}

Au fil des ans, les mauvais traitements à l'encontre de Ramandeep sont passés de la violence verbale à la violence physique et sexuelle. Durant cette période, Ramandeep a pu bénéficier d'une aide informelle et a passé une grande partie de son temps et de son énergie à élever ses enfants. Quatorze ans après leur mariage, la police a été appelée une première fois au domicile conjugal (février 2006). Aman était complètement ivre et exigeait les clés de la voiture. Ramandeep refusait de lui donner et Aman l'avait attrapé par les cheveux, l'avait poussé au 
sol et l'avait frappée à plusieurs reprises. Les enfants avaient assisté à cette agression. Ramandeep s'est alors enfui de la maison avec ses enfants et a appelé le 911. La police est arrivée à la maison. Aman a été accusé d'agression et expulsé de la maison. Il a été condamné et interdit tout communication avec Ramandeep pendant un an. La police a signalé l'incident à la Société d'aide à l'enfance (CAS). Ramandeep est resté au domicile conjugal avec les enfants. Après une séparation d'un an, Aman est retourné au domicile conjugal en faisant des excuses et en payant une pension alimentaire, mais Ramandeep a refusé de le recevoir dans sa chambre. Peu après son retour, Aman a expliqué à Ramandeep qu'il souhaitait envoyer leur fils de six ans vivre en Inde chez ses parents. Ramandeep a accepté à contrecœur et leur fils est resté en Inde pendant deux années. Les agressions verbales et physiques se sont poursuivies et Aman exigeait des rapports sexuels quotidiens. Leur fils est revenu au Canada deux plus tard, en mai 2009. Témoins des nombreuses scènes de violence, les deux enfants avaient peur de leur père et se cachaient pour l'éviter. En mai 2009, la police a été appelée une nouvelle fois. Cette fois-ci, aucune charge n’a été retenue contre Aman, mais la SAE a été saisie.

En décembre 2009, après cet incident, la police a été appelée à nouveau : Ramandeep, maintenant âgée de 39 ans, avait été battue à poing fermé et à plusieurs reprises par Aman. Elle n'avait pas préparé le diner et elle avait reçu un hématome sur la joue en guise de punition. Les enfants étaient présents et ont appelé le 911 à la demande de leur mère. Aman a été expulsé du domicile, inculpé et condamné pour voies de fait. Il a été déclaré coupable par le tribunal pénal et s'est vu interdit toute communication avec Ramandeep pendant une période d'un an, à partir de décembre 2010. Mais Aman n'a pas respecté l'ordonnance. À partir d'avril 2011, il a commencé à appeler Ramandeep pour l'agresser verbalement à chaque fois qu'il était ivre.

Peu après le dernier incident, Ramandeep a demandé de l'aide à un avocat. Craignant pour sa sécurité, elle a demandé une ordonnance d'éloignement au tribunal de la famille. Elle a aussi sollicité une ordonnance de nondéplacement pour ses enfants, car elle redoutait qu'Aman ne tente de les faire sortir du Canada. Ramandeep a réclamé la garde exclusive de ses enfants, car c’était elle qui s'était toujours occupée toute seule de la quasitotalité de leurs besoins. Elle a accepté, voire même souhaité, que ses enfants passent du temps en compagnie de leur père, à condition que celui-ci ne boive pas ni ne fume en leur présence. Ramandeep a déposé une demande de pension alimentaire rétroactive pour ses enfants, à compter de la date de la séparation avec Aman et sur la base du revenu actuel d'Aman, conformément aux Lignes directrices fédérales sur les pensions alimentaires pour enfants. Tout au long de la procédure judiciaire, Aman a fait preuve d'une certaine opacité à propos de ses biens (propriété, REER et assurance). Ramandeep s'est retrouvée dans une situation financière difficile, car elle avait dû s'endetter pour s'occuper de ses enfants. Des conflits sporadiques ont éclaté entre les enfants, ce qui a conduit à une occasion au placement du fils par la Société d'aide à l'enfance (SAE). Mais le fils a été confié de nouveau aux soins de Ramandeep.

\section{Résolution du problème}

À l'issue de trois années de procédure, Ramandeep a obtenu la garde exclusive des enfants et Aman a été condamné à payer une pension alimentaire. Le domicile conjugal a été vendu et le produit de la vente a été partagé également entre les deux parties. Aman a payé ses arriérés de pension alimentaire pour les enfants avec 
l'argent reçu de la vente. Ramandeep a accordé à Aman un droit de visite limité aux enfants, estimant que la relation entre le père et les enfants était bénéfique pour ceux-ci. Mais Aman a rejeté ses enfants et ne leur rend pas visite. Il invoque les troubles de comportement de son fils comme motif de sa décision. Le fils participe à un programme de counseling, notamment pour l'aider à surmonter le rejet de la part de son père. 


\section{ÉTUDE DE CAS 2 : ZAKIA ET WASIM}

\begin{tabular}{|c|c|c|}
\hline Profil & Femme & Homme \\
\hline Nom & Zakia & Wasim \\
\hline Âge au moment du mariage & 18 & 19 \\
\hline Âge* & 23 & 24 \\
\hline Pays d'origine & Afghanistan & $\begin{array}{l}\text { Afghanistan Wasim avait le statut de } \\
\text { réfugié en Inde avant de venir au } \\
\text { Canada. }\end{array}$ \\
\hline Religion & Musulman & $\begin{array}{l}\text { Musulmane à la naissance. Converti } \\
\text { au christianisme. }\end{array}$ \\
\hline Éducation & $\begin{array}{l}\text { Onzième année d'études complète en } \\
\text { Afghanistan. Cours supplémentaires } \\
\text { en Inde }\end{array}$ & Douzième année d'études en Inde \\
\hline Connaissance de l'anglais & Limitée & Limitée \\
\hline Emploi avant la migration & Aucune information disponible & Aucune information disponible \\
\hline Emploi & Vendeur & Vendeur \\
\hline Catégorie d'immigration & $\begin{array}{l}\text { Réfugié au sens de la convention. } \\
\text { Programme de parrainage } \\
\text { communautaire (2016) }\end{array}$ & $\begin{array}{l}\text { Réfugié au sens de la convention. } \\
\text { Programme de parrainage } \\
\text { communautaire (2016) }\end{array}$ \\
\hline Statut d'immigrant* & Résident permanent & Résident permanent \\
\hline $\begin{array}{l}\text { Nombre d'années de mariage } \\
\text { Enfants* : } \\
\text { - Fille : Abida ( } 1 \text { an })\end{array}$ & & \\
\hline
\end{tabular}

*Au moment de la demande auprès de la Cour de la famille

\section{Parcours avant la migration}

Zakia a épousé Wasim en Inde, en janvier 2012. Elle avait dix-huit (18) ans. Zakia et Wasim vivaient tous 
les deux chez les parents de Wasim, sous le statut de réfugié en Inde. Ils étaient titulaires d'une carte d'identité de réfugié du HCR. Bien qu'ils soient tous deux d’origines musulmanes, Wasi s’était converti au christianisme pendant son séjour comme réfugié en Inde. Pour pouvoir épouser Zakia, Wasim s'est reconverti à l'Islam au moment du mariage. C'était un premier mariage et Zakia croyait qu'il s'agissait d'un mariage d'amour. En Inde, Zakia et Wasim travaillaient dans des emplois mal rétribués qui leur permettaient juste de survivre. Le lendemain de leur mariage, la belle-mère de Zakia, une avocate, a persuadé sa belle-fille de signer un contrat stipulant qu'en cas de divorce, elle ne recevrait aucune compensation. Une semaine après leur mariage, Wasim a commencé à tromper Zakia. Ce comportement s'est répété tout au long de leur mariage.

Quatre ans après leur mariage, Wasim a annoncé à Zakia qu'il s'était converti de nouveau au christianisme. La famille de Wasim infligeait à Zakia des violences psychologiques permanentes et insultait sa famille et l'Islam. Le père de Wasim jetait les articles religieux de Zakia en les qualifiant de « conneries ». Un jour, Wasim a agressé physiquement Zakia : il l'a saisi par le cou et l'a frappé sur la bouche, provoquant un œedème qui a empêché Zakia de manger pendant une semaine. Zakia a été victime à nombreuses reprises des violences physiques exercées par Wasim. À une occasion, elle avait contacté les autorités, mais celles-ci lui avait dit que sa demande d'asile pourrait être compromise et elle avait renoncé à déposer une plainte officielle.

Zakia devait reverser tous ses revenus à la famille de Wasim en échange du loyer et de la nourriture. Après avoir accepté cette situation pendant un certain temps, elle a insisté pour conserver une partie de son salaire, ce qui a provoqué la colère de sa belle-famille et empiré la situation.

Zakia est tombée enceinte trois fois. À chaque fois, la famille de Wasim l'a obligée à avorter, en prétextant qu'il serait trop coûteux d'avoir des enfants. Sans autre choix, elle s'est résignée, a subi la perte des enfants et a éprouvé les douleurs physiques de l'avortement.

Avant le mariage, la famille de Wasim avait promis à Zakia qu'elle pourrait retourner à l'école pour terminer ses études. Après le mariage, elle l'a dissuadé de le faire en affirmant que cela couterait trop cher et qu'elle serait entourée d'un trop grand nombre d'hommes. Malgré cela, Zakia est retournée à l'école, mais a dû redoubler sa onzième année d'études, car sa onzième année en Afghanistan n'a pas été reconnue. Mais elle n’a pas pu terminer sa douzième année, car elle s'est installée au Canada avec son conjoint et sa belle-famille.

\section{Installation au Canada}

Zakia, Wasim et sa famille élargie sont arrivés au Canada le 26 octobre 2016, en tant que résidents permanents. Ils ont immigré en tant que réfugiés parrainés par un groupe privé au sens de la Convention outrefrontière. Au Canada, le couple a vécu avec les parents et les deux frères de Wasim. Le couple a trouvé un travail dans la même entreprise et Wasim a commencé à tromper sa femme avec plusieurs collègues de travail. Wasim et sa famille ont continué à prélever l'argent de Zakia et à contrôler ses activités quotidiennes, notamment sa tenue vestimentaire, son alimentation et ses amis. En novembre 2016, Zakia a découvert qu'elle était de nouveau enceinte. Sa belle-famille a fait pression sur Zakia pour qu'elle avorte, mais celle-ci a refusé. Elle a dû acheter elle-même les vêtements pour la maternité et engager d’autres dépenses. 


\section{Violence domestique}

Outre le fait de ne plus pouvoir pratiquer sa foi, Zakia a subi des violences physiques, psychologiques et financières et elle a été victime d'un abandon matériel et moral. Les différences de religion entre les familles de Zakia et Wasim étaient une source de discorde importante. Elle devait rembourser ses beaux-parents lors des sorties et des excursions. Elle n'était pas autorisée à envoyer de l'argent à sa famille en Inde. Elle ne pouvait pas choisir ses amis sans le consentement de sa belle-famille. La famille de Wasim surveillait de près la nourriture qu'elle mangeait, les endroits qu'elle fréquentait et les vêtements qu'elle portait. Elle n'était pas autorisée à poursuivre ses études. Un soir d'hiver, alors qu'elle était enceinte de quatre (4) mois, Zakia a demandé à Wasim de l'emmener faire des courses. Il l'a accompagné à l'épicerie, mais l'a abandonnée sur place, la forçant à rentrer chez elle à pied par $-27^{\circ} \mathrm{C}$. Lorsqu'elle est finalement arrivée à la maison à $22 \mathrm{~h} 30$, elle a découvert que Wasim et sa famille avaient fini le repas, sans lui laisser la moindre nourriture. Après avoir salué sa bellefamille, elle est entrée dans la cuisine pour préparer quelque chose à manger. Sa belle-famille a alors commencé à la réprimander en lui reprochant de ne pas les avoir salués. Elle leur a expliquait qu’elle les avait salués, qu'elle avait faim, qu'elle était fatiguée et qu'elle voulait manger quelque chose. Son beau-père l'a traité de « sale musulmane », a insulté sa famille et sa religion. Son beau-père lui a dit qu'elle vivait dans leur maison et non dans la sienne et a exigé qu'elle parte.

Zakia a pris les paroles de son beau-père au sérieux, compte tenu de la nature violente de la famille, qu'elle avait pu observer au fil des ans. Quelques semaines auparavant, elle avait vu le cousin de Wasim agresser sa sœur, ce qui avait nécessité une intervention médicale. Zakia a alors décidé de partir pour se protéger et protéger le bébé qui allait naître. Elle est partie immédiatement au milieu de la nuit. On était en plein hiver, mais Zakia, effrayée, était déterminée à ne pas revenir. Elle n’avait que 20 \$ en poche et une carte de crédit. Un Uber l’a emmené à un Holiday Inn où elle est restée une nuit. Le lendemain, en arrivant au travail, elle a reçu un texto de son conjoint l'informant qu'il ne la considérait plus comme sa femme. Il invoquait le divorce en citant à trois reprises le mot «talak », ce qui constitue un divorce selon la loi islamique. Le couple s'est séparé ce jourlà et Wasim a dit qu'il allait envoyer les papiers du divorce. Les collègues de Zakia lui ont conseillé d'appeler le 911, ce qu'elle a fait. La police a recueilli des renseignements préalables et l'a emmené dans un refuge. Peu de temps après, Zakia a signalé les mauvais traitements qu'elle avait subis à une infirmière de santé publique qui l'a orienté vers la Société de l'aide à l'enfance, car elle était préoccupée par le bien-être de l'enfant à naître.

Zakia et Wasim ont continué à se voir au travail, mais sans interagir. Sur recommandation de sa sage-femme, Zakia a dû interrompre son travail à cause de sa santé. Wasim refusait toujours de lui parler ou de s'informer sur sa santé ou celle du bébé. Zakia a accouché en urgence de sa fille par césarienne Wasim lui a rendu visite une seule fois après l’accouchement, en compagnie de ses collègues de travail.

\section{Résolution}

Zakia s'est vu confier la garde exclusive de sa fille, qui réside actuellement dans un logement de l'État. Wasim est autorisé à rendre visite à sa fille, mais les déplacements sont organisés par une tierce partie. Wasim paie une 
pension alimentaire mensuelle, mais il ne voit plus ni ne contacte sa fille depuis février 2019. Zakia s'est inscrite à l'école pour terminer ses études secondaires. 


\section{ÉTUDE DE CAS 3 : MIREMBA ET JAMES}

\begin{tabular}{|c|c|c|}
\hline Profil & Femme & Homme \\
\hline Nom & Miremba & James \\
\hline Âge au moment du mariage & 25 & 37 \\
\hline Âge* & 32 & 44 \\
\hline Pays d'origine & Ouganda & $\begin{array}{l}\text { Ouganda, résidence et travail au } \\
\text { Canada }\end{array}$ \\
\hline Religion & Chrétienne & Chrétienne \\
\hline Éducation & Enseignement secondaire & $\begin{array}{l}\text { Diplôme en gestion de chaine } \\
\text { d'approvisionnement }\end{array}$ \\
\hline Connaissance de l'anglais & Bon niveau & Bon niveau \\
\hline Emploi avant la migration & Étudiant & Aucune information disponible \\
\hline Emploi & Sans emploi & $\begin{array}{l}\text { Emploi à temps plein en tant que } \\
\text { chef d'équipe d'une grande chaine } \\
\text { d'épicerie }\end{array}$ \\
\hline Catégorie d'immigration & $\begin{array}{l}\text { Parrainage familial : James a parrainé } \\
\text { Miremba dans le cadre du } \\
\text { programme de parrainage des } \\
\text { conjoints en } 2006 \text {. }\end{array}$ & Aucune information disponible \\
\hline Statut d'immigrant* & Résident permanent/citoyen & Résident permanent/citoyen \\
\hline \multicolumn{3}{|c|}{$\begin{array}{l}\text { Nombre d'années de mariage* : } 6 \\
\text { Enfants* : }\end{array}$} \\
\hline
\end{tabular}

*Au moment de la demande auprès de la Cour de la famille

\section{Parcours avant la migration}

Miremba vivait et étudiait en Ouganda quand elle a rencontré James par l'intermédiaire d'un membre de sa famille. James habitait au Canada. Miremba et James se sont mariés en Ouganda le 10 juin 2005. C'était un premier mariage pour tous les deux. Ce n'est qu'après leur mariage que Miremba a découvert que James avait 
un enfant né d'une relation antérieure. James exerçait un emploi bien rémunéré et à temps plein comme chef d'équipe dans une grande chaine d'épicerie. Il a parrainé Miremba pour qu'elle puisse immigrer au Canada. Elle est arrivée en mars 2006, six mois après leur mariage. Elle a emménagé au domicile de James, une maison avec trois chambres dans une grande ville du Canada.

\section{Installation au Canada}

Immédiatement après son arrivée au Canada, la vie de Miremba a été bouleversée par la conduite singulière de James et de ses amis, leur consommation d'alcool et leurs fêtes. James avait l'habitude d'aller dans des clubs et de ramener des amis à la maison pour faire la fête jusqu'aux premières heures du matin. Quelques mois après son arrivée, elle est tombée enceinte et a donné naissance à sa fille. Lorsque Miremba a demandé de l'argent à James pour s'acheter des vêtements de maternité, celui-ci a refusé de lui en donner et Miremba a été obligé de porter des vêtements sans pouvoir fermer les fermetures Éclair ou les boutons.

\section{Violence domestique}

Tout au long de leurs six années de mariage, la relation conjugale a été marquée par les mauvais traitements. Après la naissance de leur fille, James a continué à amener des amis pour boire et faire la fête à la maison. À plusieurs reprises, les voisins ont appelé la police pour se plaindre de la musique et des bagarres. Mais la police n’arrivait jamais à attraper les amis de James, car ceux-ci partaient avant l'arrivée des policiers. Le weekend, Miremba devait nettoyer la maison après les fêtes. James hébergeait souvent des gens durant de longues périodes, dont certains étaient devenus de véritables locataires.

Lorsque Miremba a commencé à travailler à temps partiel dans une friperie et que James devait s'occuper de leur fille, celui-ci se montrait souvent négligent. Il lui arrivait de laisser le bébé chez son cousin ou un locataire de la maison. Un jour, Miremba est rentré à la maison pour découvrir que les bras et les jambes de sa fille d'un an étaient très enflés. Elle a alors emmené sa fille en urgence à l'hôpital où celle-ci est restée une semaine. James a refusé de l'aider en prétextant que ce n'était pas sa responsabilité. Durant toute la maladie de leur fille, James n'a pas pris un seul jour de congé pour s'occuper de sa fille. C'est Miremba qui a dû prendre deux semaines de congé pour le faire. Le cousin de James a apporté une aide pendant la convalescence de l'enfant. Quand ils ont décidé d'avoir d'autres enfants, Miremba et James ont eu une longue discussion sur la nécessité pour James de changer de comportement. Il était d'accord. Lorsque Miremba est tombée enceinte de leurs fils, James était content. Quelques jours plus tard, James a amené une femme qui devait habiter avec eux et Miremba les a trouvés en train de boire ensemble. Miremba a alors appelé le cousin de James pour lui demander de parler avec James à ce sujet.

Un jour, Miremba était allé aux funérailles d'une femme de la communauté qui avait été assassinée par son ex-mari. En rentrant chez elle, elle a découvert James et ses amis en train de boire et de crier devant la télévision. L'un des amis de James s'est alors approché de Miremba pour la menacer en lui disant qu'elle devrait recevoir une leçon comme la femme assassinée. Miremba a eu peur et a demandé à James d'intervenir. L’ami de James a continué à la menacer en affirmant qu'il allait venir tous les jours à la maison, même si cela ne lui plaisait 
pas. Comme de nombreux amis de James avaient un casier judiciaire et qu'ils étaient recherchés par la police, Miremba était inquiète pour sa sécurité. À la même époque, James a commencé une relation extraconjugale. Un jour, il a amené sa petite amie à un concert organisé pour la communauté ougandaise, une situation très embarrassante pour Miremba.

Lorsque le fils de Miremba est né en décembre 2009, James n’a pas pris un seul jour de congé pour l'aider. Il a continué à boire sans modération et à faire la fête, alors qu'elle devait s'occuper de toutes les tâches ménagères et des deux enfants. Quand il aidait, il se comportait de manière irresponsable. Un jour, en emmenant son fils au parc, il a perdu la poussette du bébé, après avoir bu de la bière.

James n'apportait aucun soutien financier à Miremba qui devait lutter pour joindre les deux bouts. Elle a alors dû emprunter de l'argent (auprès d'un ami et d'un locataire) pour suivre un cours de préposé aux services de soutien à la personne (PSP), afin d'améliorer la situation financière de la famille. James ne subvenait plus aux besoins de sa famille (pas d'argent pour la nourriture et les couches), car son emploi était devenu sporadique.

En 2011, Miremba a trouvé un emploi à temps partiel de préposée aux services de soutien à la personne. Les horaires de travail étaient en soirée. James a alors proposé de changer son service de nuit pour un service de jour afin de s'adapter aux nouveaux horaires de travail de sa femme. Mais une semaine plus tard, James est retourné à son service de nuit. Il laissait les enfants chez son cousin et Miremba venait les chercher à 23h30, ce qui n'était pas sans conséquence pour leur fille qui était toujours fatiguée et somnolente à l'école.

En 2011, six ans après leur mariage, James a été arrêté après avoir agressé sa petite amie durant une fête. À l'époque, il avait déjà dit à Miremba qu'il voulait se séparer d'elle. Elle lui avait alors demandé s'il serait intéressé par des séances de counseling, mais il avait refusé. James a alors poussé Miremba à signer des papiers concernant le refinancement de la maison, ce qu'elle n’a appris que plus tard. Une rencontre a été organisée pour que le pasteur de la communauté et sa femme assistent officiellement à la séparation de Miremba et de James. Au début de l'année 2012, Miremba et ses enfants sont allés vivre dans un refuge. Ils avaient été obligés de quitter le domicile conjugal, car James prévoyait de revenir définitivement en Ouganda et voulait vendre la maison. Bien que Miremba ait informé James de son déménagement, elle a reçu un appel de la police pour vérifier si elle vivait effectivement dans le refuge. En effet, James avait signalé la disparition de Miremba et des enfants à la police.

\section{Résolution}

Une longue bataille judiciaire a alors été engagée : elle a duré six ans. James ne voulait pas subvenir aux besoins de Miremba et de ses enfants. Le jugement final a accordé à Miremba la garde complète des enfants et un droit de visite à James. Miremba a obtenu une ordonnance de non-communication à l'exception de l’organisation des droits de visite. La retraite de James a été divisée. Le domicile conjugal a été vendu et Miremba a reçu sa part du produit de la vente. Miremba vit actuellement avec ses enfants dans un logement à loyer modéré et travaille comme préposée aux services de soutien à la personne dans un hôpital. Miremba souhaite acheter une maison au Canada. 
5.

ÉTUDE DE CAS 4 : SONALI ET RAVI

\begin{tabular}{|c|c|c|}
\hline Profil & Femme & Homme \\
\hline Nom & Sonali & Ravi \\
\hline Âge au moment du mariage & 27 & 29 \\
\hline Âge* & 31 & 33 \\
\hline Pays d'origine & Inde & Inde \\
\hline Religion & Hindou & Hindou \\
\hline Éducation & Diplômes M.A. et M.B.A. en Inde & Aucune information disponible \\
\hline Connaissance de l'anglais & Bon niveau & Bon niveau \\
\hline Emploi avant la migration & $\begin{array}{l}\text { Stagiaire comme cadre dans les } \\
\text { ressources humaines }\end{array}$ & $\begin{array}{l}\text { Administrateur unique et actionnaire } \\
\text { d'une société de fret automobile }\end{array}$ \\
\hline Emploi & $\begin{array}{l}\text { Travail à temps partiel dans une } \\
\text { pharmacie depuis le 18/03/2016. }\end{array}$ & $\begin{array}{l}\text { Administrateur unique et actionnaire } \\
\text { d'une société de fret automobile }\end{array}$ \\
\hline Catégorie d'immigration & $\begin{array}{l}\text { Catégorie du regroupement familial. } \\
\text { Ravi a parrainé Sonali dans le cadre } \\
\text { du programme de parrainage des } \\
\text { époux assorti d'une période } \\
\text { conditionnelle de } 2 \text { ans. (2015) }\end{array}$ & Aucune information disponible \\
\hline Statut d'immigrant* & Résident permanent & Citoyen \\
\hline $\begin{array}{l}\text { Nombre d'années de mariage } \\
\text { Enfants* : } \\
\text { - Aucun }\end{array}$ & & \\
\hline
\end{tabular}

*Au moment de la demande auprès de la Cour de la famille

\section{Parcours avant la migration}

Sonali avait vingt-sept ans et Ravi avait vingt-neuf ans lorsqu'ils se sont mariés. À cette époque, Sonali vivait en Inde. Ravi était citoyen canadien et vivait dans une grande ville de l'Ontario. Il travaillait à plein temps 
dans l'industrie automobile. En plus de son emploi, Ravi possédait une microentreprise de fret automobile. Le mariage arrangé a eu lieu en Inde en octobre 2013. C'était un premier mariage pour les deux partenaires. Sonali a continué à vivre en Inde pendant deux ans après leur mariage, car Ravi a tardé à faire la demande de parrainage de conjoint qui aurait permis à Sonali d'immigrer au Canada. Sonali est arrivée au Canada en octobre 2015 à titre de résidente permanente, assujettie à une période conditionnelle.

\section{Installation au Canada}

À son arrivée au Canada, Sonali a vécu avec Ravi et la famille de celui-ci (parents et deux sœurs) dans une maison appartenant à Ravi, son père et ses sœurs. À cette époque, la famille de Ravi tenait des propos agressifs à l'égard de Sonali. Sa belle-mère interférait de façon inappropriée dans la vie du couple, leur interdisant ainsi toute intimité. La belle-mère avait l'habitude de venir dans leur chambre et d'insister pour dormir entre les deux époux. Un jour, elle a fait irruption dans la salle de bains alors que le couple se douchait ensemble.

\section{Violence domestique}

Sonali a été victime de mauvais traitements de la part de Ravi et de sa belle-famille. Sa belle-mère l'agressait verbalement et physiquement. Un jour, alors que Sonali téléphonait à sa mère en Inde, sa belle-mère a commencé à écouter la conversation sur une autre ligne. Lorsque Sonali a demandé des explications à sa bellemère, celle-ci s'est fâchée et a giflé Sonali. Sonali s'est mise à pleurer et a téléphoné à Ravi sur son lieu de travail. Celui-ci est rentré à la maison pour emmener Sonali à son bureau. Après cet incident, Sonali accompagnait Ravi tous les jours à son bureau, car elle ne voulait plus se retrouver toute seule avec sa belle-mère. Avec le temps, sa belle-mère et ses belles-sœurs ne lui adressaient plus la parole.

Un jour d'hiver, le couple était en voiture et Ravi a commencé à se disputer avec Sonali en criant et en claquant le volant avec sa main. Il lui a ordonné de sortir de la voiture et l'a déposée sur le bord de la route. Sonali a demandé de l'aide à un couple. Le couple a accepté de l'aider et l'a déposée à un poste de police. La police a enquêté et a ramené Sonali au domicile conjugal. Les policiers ont parlé avec la belle-mère de Sonali qui a déclaré qu'elle n’avait aucun problème à ce que Sonali vive avec eux. Les agents de police ont donné à Sonali la possibilité d’aller dans un refuge. Sonali n’a pas voulu se séparer de son mari et décidé de se rendre au bureau de Ravi. Finalement, elle est retournée au domicile conjugal.

Les tensions ont continué à augmenter et une autre dispute a éclaté entre Sonali et sa belle-mère. Sonali s'était absenté de la maison pendant un moment et sa belle-mère avait enfermée pour la punir. Après cet incident, Sonali et Ravi ont décidé de quitter la maison familiale. Ils ont obtenu l'autorisation de l'employeur de Ravi de rester trois nuits dans le bureau de Ravi. Ils n'avaient pas de vêtements de rechange et mangeaient dans un temple local. Finalement, l'employeur de Ravi les a emmenés chez lui et leur a permis d'utiliser les installations de sa maison. Il leur a conseillé de déménager pour commencer une nouvelle vie.

En mars 2016, Sonali et Ravi ont emménagé dans un appartement de copropriété. Ravi avait acheté ce bien avant son mariage, mais le louait pour en tirer un revenu. Sonali a commencé à travailler à temps partiel dans une grande chaine de pharmacies, car elle ne recevait pas d'argent de la part de Ravi. 
Ravi lui a demandé d'acheter tous les meubles nécessaires à l'appartement (lit, matelas, etc.) avec son argent personnel. Sonali était venue au Canada avec très peu d'argent. Une fois cette somme dépensée, sa mère lui envoyait de temps en temps de l'argent depuis l'Inde. Sonali devait se charger des dépenses d'alimentation et de ses factures de téléphone.

Sonali a été victimes de violences verbales, physiques, financières et sexuelles de la part de son mari. Celuici était imprévisible et sujet à des sautes d'humeur violentes. Il se mettait en colère pour des motifs difficiles à comprendre pour Sonali. Il buvait de façon excessive et avait souvent des comportements étranges. Ainsi, il lui arrivait de sortir en voiture avec Sonali, de la déposait à un endroit au hasard et de la signalait comme disparue à la police. À de nombreuses occasions, Ravi a agressé physiquement Sonali. Au printemps 2016, à la suite d'une agression qui lui avait laissé une ecchymose au bras, Sonali a consulté son médecin de famille et lui a expliqué qu'elle avait été maltraitée. Le médecin lui a conseillé de signaler le mauvais traitement à la police et de se rendre dans un refuge, mais Sonali a refusé en affirmant qu'elle voulait essayer de sauver son mariage. Peu de temps après, Ravi l’a agressée de nouveau, lui occasionnant une blessure ouverte au bras. Ravi a accompagné Sonali aux urgences de l'hôpital et l'a obligé de dire aux médecins qu'elle s'était coupée en faisant la vaisselle. Sonali a reçu plusieurs points de suture. Le comportement de Ravi était erratique. Il agissait ou s'exprimait de façon blessante à son égard, puis s'excusait. Au cours d'une sortie dans un centre commercial, Ravi s'est approché d'un policier en déclarant que Sonali était mentalement instable et suicidaire et qu'elle s'était lacérée elle-même. Sonali a parlé avec les policiers pour expliquer la situation. Les policiers ont demandé à Sonali s'ils devaient arrêter et inculper son conjoint, mais celle-ci a répondu que non. Les policiers ont ramené Sonali à son appartement. Ravi est alors resté quelques nuits chez ses sœurs avant de retourner à l'appartement.

En janvier 2017, le couple s'est rendu en Inde pour assister à un mariage et rendre visite à sa famille. Lors du mariage, Ravi s'est saoulé et a insulté Sonali devant les membres de sa famille. Lors d'un voyage dans un pays voisin, Ravi s'est mis en colère : il a exigé le passeport de Sonali et lui a demandé de ne pas retourner avec lui au Canada. Puis de retour à l'endroit où ils séjournaient, il a agi comme si de rien n'était. Sonali était de plus en plus inquiète du comportement erratique de Ravi.

À son retour au Canada, Ravi a perdu son emploi. Peu de temps après, il a agressé Sonali, mais cette fois, celle-ci a appelé le 911. Ravi a été inculpé de trois chefs d'accusation pour agression. Il a été libéré sous caution avec interdiction de la contacter directement ou indirectement ou de se rendre au domicile conjugal. Malgré cela, sa belle-mère a tenté à de nombreuses reprises de la contacter pour qu'ils se réconcilient. En mai 2018, Ravi est passé devant la Cour pénale où il a plaidé coupable des accusations portées contre lui. Craignant pour sa vie, Sonali a demandé une ordonnance d'éloignement. En raison de ces mauvais traitements, Sonali est aujourd'hui victime de crises d'anxiété et de stress et elle a du mal à se concentrer. Elle éprouve des difficultés à faire confiance et à nouer des relations. Elle souffre également de maux de tête qui affectent sa vie quotidienne.

Lors de la procédure de séparation, Ravi n’a pas déclaré toute sa situation financière. Il n’a pas communiqué, en particulier, qu'il était travailleur autonome, car il ne voulait pas que ce revenu soit pris en compte dans le calcul de la pension alimentaire de son épouse. Avant le mariage, il avait contracté des dettes importantes qu'il n'était plus en mesure de rembourser. Il avait déposé une proposition de consommateur en janvier 2013, dont 
il allait payer les créances tout au long de son mariage. Sonali a découvert ces problèmes financiers au moment de leur séparation.

\section{Résolution}

L'appartement de copropriété a été vendu et Sonali a reçu une somme forfaitaire après le remboursement de l'hypothèque et des taxes. Sonali est désormais en sécurité. Elle a reçu un règlement financier significatif et une ordonnance d'éloignement de la Cour de la famille. Ravi et Sonali ont fini par divorcer. Elle exerce actuellement deux emplois et dispose d'une relative autonomie financière. Elle a pu acheter un appartement de copropriété avec l'aide en partie de sa famille en Inde. 
6.

ÉTUDE DE CAS 5 : GAGANDEEP ET KULDIP

\begin{tabular}{|c|c|c|}
\hline Profil & Femme & Homme \\
\hline Nom & Gagandeep & Kuldip \\
\hline Âge au moment du mariage & 25 & 35 \\
\hline Âge* & 27 & 37 \\
\hline Pays d'origine & Inde & Inde \\
\hline Religion & Sikh & Sikh \\
\hline Éducation & Enseignement secondaire & Enseignement secondaire \\
\hline Connaissance de l'anglais & Limitée & Limitée \\
\hline Emploi avant la migration & Aucune information disponible & $\begin{array}{l}\text { Employé dans le secteur du bâtiment } \\
\text { en Italie }\end{array}$ \\
\hline Emploi & $\begin{array}{l}\text { Emploi à temps partiel dans la } \\
\text { restauration rapide }\end{array}$ & $\begin{array}{l}\text { Emploi à temps plein dans le } \\
\text { bâtiment }\end{array}$ \\
\hline Catégorie d'immigration & $\begin{array}{l}\text { Gagandeep a immigré avec sa famille } \\
\text { au Canada, en } 2005\end{array}$ & $\begin{array}{l}\text { Regroupement familial : Gagandeep } \\
\text { a parrainé Kuldip dans le cadre du } \\
\text { programme de parrainage des } \\
\text { conjoints en } 2009 \text {. }\end{array}$ \\
\hline Statut d'immigrant* & Citoyen & Résident permanent \\
\hline \multicolumn{3}{|c|}{$\begin{array}{l}\text { Nombre d'années de mariage* }: 2 \\
\text { Enfants* : } \\
\text { - Fils : Ravi : (11 mois, né avec de nombreux problèmes de santé. Il a subi une chirurgie majeure en bas âge. Il doit } \\
\text { recevoir des médicaments et des soins continus.) }\end{array}$} \\
\hline
\end{tabular}

*Au moment de la demande auprès de la Cour de la famille

\section{Parcours avant la migration}

Gagandeep a immigré avec ses parents au Canada en 2005. Elle s'est mariée à Kuldip en 2008. Leur mariage a été arrangé par leurs parents. Ceux-ci se sont rencontrés grâce à une annonce matrimoniale dans un journal 
local. Le mois suivant, le mariage était célébré selon les rituels religieux sikhs. Avant son mariage, Kuldip vivait en Italie où il travaillait dans le bâtiment, alors que sa mère et sa sœur résidaient au Canada.

\section{Installation au Canada}

Après son mariage, Kuldip s'est installé au Canada avec un visa de visiteur et Gagandeep a déménagé pour vivre avec la famille élargie de son mari. Gagandeep a parrainé l'immigration de Kuldip au titre du programme de parrainage des conjoints et, un an plus tard, Kuldip a obtenu son statut d'immigrant reçu. Pendant les premiers mois de leur mariage, aucun incident ne s'est produit. Gagandeep était employé à plein temps dans un restaurant et Kuldip travaillait dans le bâtiment. Gagandeep est tombée enceinte peu de temps après leur mariage et leur fils est né en janvier 2009.

\section{Violence domestique}

Moins d'un an après le mariage, leur relation s'est dégradée avec la naissance du fils de Gagandeep. Elle a empiré lorsque Kuldip a obtenu son statut d'immigrant reçu. Immédiatement après l'accouchement par césarienne, Kuldip a imposé à Gagandeep une relation sexuelle. Gagandeep a dû recourir à des soins médicaux.

En avril 2009, lorsque Kuldip a obtenu son nouveau statut d'immigrant, il a commencé à maltraiter verbalement et physiquement Gagandeep et son bébé. Le couple possédait un compte bancaire commun que Kuldip s'est employé à vider. Il a ouvert parallèlement son propre compte bancaire. Kuldip a accusé Gagandeep de manquer de respect à l'égard de sa mère et l'a frappé pour la punir. Il lui a demandé de quitter la maison avec son bébé. Leur fils a eu de nombreux problèmes de santé et a subi une intervention chirurgicale à l'âge de 3 mois. Kuldip n’a contribué à aucun des examens médicaux importants requis pour assurer le suivi.

Les mauvais traitements sont devenus plus violents. En avril 2009, Kuldip a giflé Gagandeep si fort que celleci est tombée sur le sol. Kuldip l’a saisi par les cheveux pour la frapper à la tête et la pousser contre le mur. Il lui a donné un coup de pied dans l'estomac et dans le dos.

En juin 2010, Kuldip est sorti pour boire. Quand il est revenu, il a exigé d’avoir des rapports sexuels avec Gagandeep. Elle lui a répondu qu'elle lui obéirait après avoir mis le bébé au lit. Kuldip est devenu violent, il l'a battue et s'est jeté sur elle alors qu'elle tenait son bébé dans ses bras. Gagandeep s'est évanoui. Lorsqu'elle a repris conscience, elle a demandé de l'aide à sa belle-mère qui lui a refusé. L’incident a traumatisé le bébé qui a fait une poussée de fièvre. Le lendemain, Gagandeep a emmené le bébé chez le médecin qui a remarqué les marques sur le visage de Gagandeep : c'était les séquelles des violences subies la nuit précédente. Gagandeep n’a pas révélé à son médecin les causes réelles de ces lésions. Le même jour, Kuldip a parlé des événements de la veille avec son cousin. Celui-ci lui a conseillé de ne pas frapper Gangandeep ni de lui imposer des relations sexuelles. Gagandeep est allé chez son cousin pour y séjourner pendant quelques jours avec le bébé. Kuldip s'est rendu chez le cousin pour s'excuser auprès de Gagandeep et lui promettre qu'il ne la maltraiterait plus jamais. Elle a décidé de retourner vivre avec lui.

Mais Kuldip n’a pas tenu parole et les mauvais traitements sont devenus plus violents. Les passages à tabac et les rapports sexuels forcés se sont poursuivis, souvent avec le bébé dans les bras de Gagandeep. La colère de 
Kuldip envers le bébé s'est intensifiée. Un soir, Kuldip a ordonné à son fils de 18 mois d’arrêter de toucher ses jouets. Mais celui-ci continue à jouer et Kuldip l'a gifle au visage et a frappé Gagandeep quand celle-ci a tenté de protéger son enfant. Peu de temps après, Gagandeep a décidé de parler de ces violences avec ses parents. Ceux-ci lui ont conseillé d'essayer de sauver son mariage.

Le dernier incident est survenu lorsque Kuldip est rentré tard la nuit en état d'ivresse. Il a commencé à proférer des obscénités particulièrement blessantes et à insulter les parents de Gagandeep. Lorsque Gagandeep lui a tenu tête, Kuldip lui a cogné la tête contre la tête du lit et a commencé à l'étouffer en lui disant qu'il allait la tuer. Gagandeep a réussi à prendre son téléphone et à appeler le 911. Kuldip s'est saisi du téléphone et l'a cassé. Mais la police était déjà en route. La belle-mère de Gagandeep est alors entrée dans la pièce pour empêcher Gagandeep de sortir et a dit à son fils de la tuer. Elle lui a dit qu'ils n'avaient plus besoin d'elle, car Kuldip avait déjà obtenu son statut d'immigrant reçu. La police est arrivée avant que Kuldip ne puisse mettre les paroles de sa mère à exécution.

La police a emmené Kuldip hors de la maison et l’a arrêté pour voies de fait et menaces. Malgré la présence de sa belle-mère, Gagandeep a passé la nuit au domicile conjugal et a emménagé chez ses parents le lendemain. Kuldip a négocié sa libération sous caution avec interdiction de communiquer avec Gagandeep. En novembre 2010, après avoir reçu le soutien de conseillers et de travailleurs sociaux chargés de l'aide aux victimes, Gagandeep a accusé Kuldip d'agression sexuelle. Gagandeep a alors demandé une ordonnance d'éloignement à l'encontre de Kuldip à la Cour de la famille.

\section{Résolution}

Plus de trois ans après l'incident, Kuldip a été condamné par la Cour pénale à une mise à l'épreuve de cinq ans avec interdiction de communication avec Gagandeep pendant deux ans. Gagandeep a reçu la garde exclusive de son fils. Kuldip a obtenu un droit de visite surveillé de l'enfant. En 2016, Gagandeep et Kuldip ont divorcé. Gagandeep s'est remariée et vit avec son nouveau mari et son fils. Elle travaille à temps plein et ses parents l'aident pour s'occuper de son enfant. 


\section{ÉTUDE DE CAS 6 : NAMRATA ET DINESH}

\begin{tabular}{|c|c|c|}
\hline Profil & Femme & Homme \\
\hline Nom & Namrata & Dinesh \\
\hline Âge au moment du mariage & 22 & 28 \\
\hline Âge* & 35 & 41 \\
\hline Pays d'origine & Inde & Inde \\
\hline Religion & Jaïn & Jaïn \\
\hline Éducation & Maitrise en communication & Dentiste \\
\hline Connaissance de l'anglais & Bon niveau & Bon niveau \\
\hline Emploi avant la migration & Aucun & $\begin{array}{l}\text { Dentiste, associé au cabinet d'un } \\
\text { autre dentiste }\end{array}$ \\
\hline Emploi & Sans emploi & Dentiste, cabinet personnel \\
\hline Catégorie d'immigration & Économique & Économique (première demande) \\
\hline Statut d'immigrant* & Citoyen & Citoyen \\
\hline \multicolumn{3}{|c|}{$\begin{array}{l}\text { Nombre d'années de mariage* : } 12 \\
\text { Enfants* : } \\
\text { - Fille : Indrani (12 ans) } \\
\text { - Jumeaux : Ram et Shyam ( } 6 \text { ans) Ram et Shyam ( } 6 \text { ans). L'un des fils a des besoins spéciaux et des problèmes de } \\
\text { santé importants depuis sa naissance. Il a subi une chirurgie et nécessite beaucoup de soins. }\end{array}$} \\
\hline
\end{tabular}

*Au moment de la demande auprès de la Cour de la famille

\section{Parcours avant la migration}

Namrata et Dinesh se sont mariés en Inde où Dinesh travaillait comme dentiste. Il s'agissait d'un mariage arrangé. Les parents de Namrata et de Dinesh résidaient en Inde à l'époque. Après deux ans de vie commune, Dinesh a reçu les documents nécessaires pour pouvoir immigrer au Canada. Dinesh a déménagé au Canada sans Namrata, car celle-ci était enceinte et ne pouvait pas l'accompagner. Leur fille est née en octobre 2001, 
deux mois après le départ de Dinesh. Celui-ci était déçu, car il souhaitait avoir un garçon ${ }^{1}$ et le frère de Dinesh a manifesté son mécontentement en pressant une tasse de métal chaud sur le talon du bébé qui avait dix mois. Namrata a continué de vivre en Inde pendant dix-sept mois en élevant seule son enfant. Durant toute cette période, Namrata parlait occasionnellement à son mari. Elle a reçu ses documents d'immigration en décembre 2022 et prévoyait de rejoindre Dinesh le mois suivant, mais son beau-père a égaré son passeport et elle a été obligée de demander un passeport d'urgence pour pouvoir voyager.

\section{Installation au Canada}

Namrata a rejoint Dinesh au Canada en janvier 2003. Sa fille avait alors 15 mois. Namrata assumait l'essentiel des soins prodigués à sa fille et s'occupait de toutes les tâches ménagères. Peu de temps après l'arrivée de Namarata au Canada, Dinesh a été admis en faculté de médecine dentaire et a entrepris une formation pour pouvoir exercer comme dentiste au Canada. Dinesh consacrait tout son temps à ses études et passait très peu de temps avec sa famille. Deux ans plus tard, Dinesh a obtenu les titres nécessaires pour exercer sa profession et la famille a déménagé dans une autre ville du Canada où Dinesh travaillait comme associé dans une clinique dentaire. Namrata est tombée enceinte de ses deux fils. À sept mois, elle a été transportée d'urgence à l'hôpital à la suite de complications. Dinesh était trop occupé à la clinique dentaire pour s'occuper de sa femme. Après la naissance des jumeaux en juillet 2006, les choses sont devenues plus difficiles pour le couple. L'un de leurs fils était gravement malade et a été transféré immédiatement dans un hôpital pédiatrique pour y subir une intervention chirurgicale. Namrata récupérait de sa césarienne et ne pouvait pas accompagner l'enfant à l'hôpital. Dinesh était réticent à prendre un congé pour s'occuper de son fils, mais a finalement accepté à contrecœur. Un an après la naissance, leur autre fils a été brûlé au 3e degré lorsque du thé chaud a été renversé sur son visage alors qu'il était gardé par sa nounou. Dans les premières années de leur vie, les deux garçons ont nécessité de nombreux soins et rendez-vous médicaux. Dinesh n’a apporté aucune aide pour répondre à leurs besoins de santé ou de transports à l'hôpital ou les cliniques. Namrata a assumé toute seule cette tâche en demandant à des amis de l’accompagner en voiture.

\section{Violence domestique}

Namrata avait déjà subi des violences psychologiques de la part de son mari avant son immigration au Canada. L'abandon psychologique dont elle était victime s'est intensifié après la migration à cause du désir

1. Dans un grand nombre de cultures, on pense qu'il est préférable d'avoir un garçon plutôt qu'une fille. Cette vision traditionnelle de la culture se perpétue dans les familles de l'Asie du Sud, qui vivent aujourd'hui en Inde et dans d'autres pays comme le Canada. Pour plus d'informations, vous pouvez consulter un travail de recherche récent sur les taux de masculinité à la naissance chez les mères de deuxième génération d’origine sud asiatique en Ontario, Canada. Cette étude rétrospective, qui s’appuie sur une cohorte représentative de la population, examine certains des problèmes et des effets de la préférence culturelle pour les garçons : https://jech.bmj.com/content/jech/ 72/11/1044.full.pdf 
de Dinesh d'accumuler toujours plus de richesse. Il contrôlait l'argent de Namrata et ne lui permettait pas de participer aux décisions concernant les finances de la famille. L’année où les jumeaux sont nés, Dinesh a ouvert un cabinet dentaire en son nom et a mis Namrata sur la liste des employés pour bénéficier d'une déduction fiscale plus importante. En parallèle, il travaillait dans une autre clinique dentaire en tant qu'associé. En 2009, il a acheté le domicile conjugal. La même année, Dinesh a utilisé la maison comme garantie pour financer d'autres investissements. Il n’a pas communiqué ces informations à Namrata, bien que certaines de ces écritures aient été enregistrées au nom de celle-ci.

En octobre 2011, Dinesh est devenu de plus en plus agressif et a accusé Namarat de ne pas être fidèle à son mariage. Il a menacé de l'expulser de la maison et de lui couper les vivres. Il a appelé le frère et les parents de Namrata en Inde pour leur dire qu'il allait la chasser de la maison. Les parents de Namrata ont appelé un ami de la famille au Canada et lui ont demandé d’aider leur fille, mais Dinesh a finalement accepté de garder Namrata après en avoir parlé avec son père. Les parents de Namrata ont fait pression sur leur fille pour qu'elle s'excuse auprès de Dinesh, car ils avaient peur que ce dernier ne la chasse. Un ami commun a conseillé au couple de suivre des séances de counseling. Dinesh a refusé, mais Namrata a commencé à assister à ces séances. Le couple a emménagé dans des chambres séparées. Les parents de Namrata et de Dinesh sont allés au Canada pour faire pression sur le couple pour qu'il se réconcilie. Namrata a accepté pour le bien des enfants. L'année suivante, Dinesh a installé des caméras de surveillance dans la maison pour contrôler les allées et venues de Namrata. Il a informé son épouse qu'il avait engagé un détective privé pour la suivre. Dinesh a commencé à transférer des sommes d'argent importantes au profit de son frère en Inde, réduisant ainsi leurs avoirs au Canada. Il a demandé à Namrata de signer des papiers pour refinancer le domicile conjugal et transférer les investissements faits au nom de sa femme vers le sien. Elle a refusé et Dinesh a eu un violent accès de colère.

Tout au long de leur mariage, Namrata et Dinesh se sont disputés sur la question de savoir qu'elle était la meilleure manière d'élever les enfants. Dinesh était partisan de la manière forte pour discipliner les jumeaux. Il effrayait ses enfants en leur montrant des images violentes à la télévision et les encourager à se battre physiquement pour résoudre les problèmes. Dinesh parlait des conflits familiaux avec sa fille de neuf ans en faisant des remarques désobligeantes sur Namrata et les grands-parents maternels et paternels. Dinesh se méfiait de Namrata et la soupçonnait d'avoir une liaison avec son ami Balwant qui séjournait souvent chez eux. Dinesh a alors décidé d'installer des caméras de vidéo à l'extérieur et à l'intérieur de la maison afin de surveiller sa femme à l'aide de son smartphone.

Namrata s'inquiétait pour sa sécurité et celle des enfants, car Dinesh surveillait chacun de leurs mouvements. Un jour, Dinesh a déclaré à Namrata, en présence de leur fille adolescente, qu'il voulait faire faire un test ADN à ses enfants, car il soupçonnait qu'il n'était peut-être pas leur père biologique.

La Société de l'aide à l'enfance (SAE) est intervenue dans les affaires de la famille à deux reprises. En 2010, l'un des jumeaux, âgé de quatre ans, a dit à son professeur que son père l'avait battu avec une batte de cricket. Namrata a démenti l'incident, car elle avait peur que les enfants soient emmenés par la SAE. Deux ans plus tard, les enfants ont déclaré à leur médecin de famille qu'ils étaient victimes de mauvais traitements. En novembre 2012, Namrata et Dinesh ont eu une violente altercation lors d'une visite d'un travailleur social de la SAE 
à leur domicile. Après le départ du travailleur, Dinesh est entré par effraction dans la chambre fermée à clé de Namrata. Il a emporté les papiers et les biens personnels de Namrata et l'a accusée d'avoir une liaison extraconjugale. Les enfants ont été témoins de la scène. Dinesh a appelé la police pour leur demander de venir à la maison. Il a déformé les faits et a accusé Namrata d'en être l'auteure. La police a demandé à Namrata de quitter la maison durant une nuit sans emmener les enfants. Namrata avait raconté aux policiers que Dinesh avait forcé les serrures de sa chambre et que la SAE avait déjà été saisie auparavant. Les enfants sont restés au domicile conjugal avec la nounou et Dinesh. N’ayant reçu aucune indication de la police sur un endroit où passer la nuit, Namrata a contacté des amis pour passer la nuit chez eux. Cette nuit a marqué la séparation officielle du couple.

Lorsque Namrata est retournée au foyer conjugal le lendemain, elle a demandé de l'aide à une organisation locale qui l’a orientée vers le Service aux victimes. Le Service aux victimes a contacté la police pour ajouter au rapport de l'incident l'exposé des événements par Namrata. La police a appelé Dinesh pour l'informer que Namrata avait mis à jour le rapport. Celui-ci s'est emporté et a menacé de renvoyer Namrata et les enfants en Inde. Namrata avait peur de contacter la police. Dinesh a poussé la nounou à démissionner et Namrata s'est retrouvée toute seule avec Dinesh et ses enfants dans la maison familiale. Bien que Namrata et Dinesh soient officiellement séparés, la famille a continué à vivre ensemble au domicile conjugal jusqu'en décembre 2013. Namrata a demandé une pension alimentaire provisoire afin de pouvoir quitter la maison de Dinesh. Une fois ce document délivré par le tribunal, Namrata a quitté le foyer pour protéger ses enfants du conflit en cours et des traumatismes psychologiques induits. Elle a loué un appartement de deux chambres pour pouvoir séjourner avec ses enfants.

\section{Résolution}

Après le départ de Namrata, Dinesh a déposé une demande auprès du tribunal pour obtenir le retour des enfants. Namrata a présenté une requête incidente pour déclarer que les enfants devaient rester sous sa garde. Le tribunal a reproché à Namrata d'avoir pris des mesures « d'auto-assistance » en quittant le domicile conjugal sous prétexte de mauvais traitements passés. Le tribunal souhaitait que Namrata obtienne d’abord une ordonnance du tribunal avant de quitter le domicile conjugal. Le tribunal a également estimé que la préoccupation de Namrata à l'égard de la sécurité des enfants était suspecte : elle était prête à fournir à Dinesh un droit de visite un week-end sur deux. Dinesh a refusé de communiquer l'intégralité de ses ressources financières et Namrata l'a poursuivi en justice pour obtenir les informations nécessaires. Il s'agissait d'évaluer ses nombreux investissements professionnels de Dinesh avec le concours de nombreux experts financiers et comptables. Finalement, Namrata a été en mesure de prouver l'importance des revenus et des actifs de Dinesh, ce qui a conduit à un règlement financier significatif entre les deux parties. Le domicile conjugal a été vendu par ordonnance judiciaire et Namrata a reçu sa part du produit de la vente. Namrata a obtenu une pension alimentaire pour les enfants et l'épouse et elle a pu acheter la maison où elle vit actuellement avec ses trois enfants. Les enfants voient leur père quand son horaire de travail le lui permet. 
8.

ÉTUDE DE CAS 7 : DEEPA ET AMOL

\begin{tabular}{|c|c|c|}
\hline Profil & Femme & Homme \\
\hline Nom & Deepa & Amol \\
\hline Âge au moment du mariage & 42 & 28 \\
\hline Âge* & 53 & 39 \\
\hline Pays d'origine & Inde & Inde \\
\hline Religion & Sikh & Sikh \\
\hline Éducation & $\begin{array}{l}\text { Diplôme de premier cycle } \\
\text { universitaire }\end{array}$ & $\begin{array}{l}\text { Diplôme de premier cycle } \\
\text { universitaire }\end{array}$ \\
\hline Connaissance de l'anglais & Bon niveau & Bon niveau \\
\hline Emploi avant la migration & $\begin{array}{l}\text { Employé de compagnie aérienne; } \\
\text { personne de cabine }\end{array}$ & Ingénieur électrique \\
\hline Emploi & Sans emploi & $\begin{array}{l}\text { Création de son entreprise de } \\
\text { camionnage en } 2009 \text {; propriétaire } \\
\text { unique et directeur de l'entreprise }\end{array}$ \\
\hline Catégorie d'immigration & Économique & Économique \\
\hline Statut d'immigrant* & Résident permanent (PR) & Résident permanent (PR) \\
\hline \multicolumn{3}{|c|}{$\begin{array}{l}\text { Nombre d'années de mariage* : } 11 \\
\text { Enfants* }^{*} \\
\text { - Fils : Amar (7 ans) } \\
\text { - Fille : Anju (7 ans) }\end{array}$} \\
\hline
\end{tabular}

*Au moment de la demande auprès de la Cour de la famille

\section{Parcours avant la migration}

Deepa travaillait comme personnel navigant d'une compagnie aérienne internationale avant son mariage avec Amol. Ils résidaient tous les deux dans une grande ville de l'Inde au moment de leur mariage. Deepa 
a utilisé ses économies pour acheter le domicile familial après leur mariage. Lorsque la famille a émigré au Canada, l’appartement a été vendu et l'argent a été utilisé par Amol pour acheter une propriété en Inde en son nom propre. Deepa a opté pour un départ volontaire à la retraite de la compagnie aérienne avant d'immigrer au Canada. Elle a encaissé ses prestations de retraite pour emporter 27 000,00 CAN au Canada. Cela représentait 1500000 roupies indiennes. Dès le début de leur relation, Amol consommait des quantités excessives d'alcool.

\section{Installation au Canada}

Le couple a fait une demande d'immigration au Canada et Amol est arrivé le premier. En juillet 2007, Amol a commencé à travailler dans le secteur du bâtiment et comme manutentionnaire en installant le nouvel environnement de la famille. Deepa est arrivé en août 2008 avec ses enfants. En 2009, Amol a démarré sa propre entreprise de camionnage : il était le propriétaire unique et seul directeur de l'entreprise. À son arrivée au Canada, Deepa a découvert qu'Amol avait une relation extraconjugale avec une autre femme. Deepa lui a demandé des explications, mais celui-ci lui a dit qu'elle n'avait pas d'autre choix que de vivre avec lui. Elle a alors décidé de rester pour le bien des enfants.

\section{Violence domestique}

Le couple a commencé à avoir de sérieuses difficultés : les deux conjoints se disputaient fréquemment au sujet de la relation extraconjugale d'Amol et de sa consommation excessive d'alcool. Amol buvait et conduisait son camion avec les enfants. Il laissait trainer les bouteilles d'alcool dans la maison. Il dissimulait l'alcool dans des bouteilles de jus de fruits : un jour, leur fille a bu accidentellement de l'alcool contenu dans l'une de ces bouteilles. Lorsqu'Amol buvait, il maltraitait verbalement et physiquement Deepa sous les yeux de ses enfants. Il criait et agressait verbalement ses enfants. Il regardait des contenus sexuellement explicites sur Internet en présence des enfants. Cette conduite effrayait ses enfants. À plusieurs reprises, Amol a conduit en état d'ébriété après s'être disputé avec Deepa. À son retour, il s'est endormi dans la cour du domicile conjugal.

En septembre 2009, Amol a agressé Deepa, qui a décidé de signaler l'incident au poste de police local. Amol a été inculpé de voies de fait et du chef de menaces de mort. Il a été libéré sous caution avec interdiction de communiquer avec Deepa pendant toute la durée de la procédure judiciaire. La Société de l'aide à l'enfance (SAE) a été saisie de l'affaire. Les deux parties ont alors vécu séparément dans des appartements au sous-sol : Deepa avec les enfants et Amol tout seul. Malgré l'ordonnance de non-communication, Amol s'est rendu à plusieurs reprises au domicile conjugal pour communiquer avec ses enfants. En avril 2010, la Cour pénale a demandé à Amol de signer un engagement à ne pas troubler l'ordre public pendant douze mois avec interdiction de communication d'un an. Les familles élargies des deux parties sont intervenues pour persuader le couple de reprendre sa relation conjugale. À la demande de sa famille, Deepa s'est réconciliée avec Amol. Toutefois, la relation était fragile et Amol a fait appel à un avocat pour conditionner la réconciliation et la reprise d'une vie commune à la signature d'un accord de séparation. Amol a engagé un autre avocat pour donner des conseils juridiques soi-disant indépendants à Deepa et pour l'assister lors de la signature de l'accord. En juin 2020, Deepa a signé les documents officiels de séparation sans bien en comprendre la portée, car aucun 
des deux avocats ne lui avait fourni une explication appropriée. L'accord prévoyait le paiement symbolique d'une pension alimentaire de 150 \$ pour les enfants et dispensait Amol de l'obligation de payer une pension alimentaire à son épouse. Amol n’a pas communiqué sa situation financière lors de la signature de cet accord. L'accord prévoyait aussi que Deepa aurait la garde exclusive des enfants avec un droit de visite d'Amol à la résidence de Deepa. Deepa avait également renoncé à tous ses droits sur la propriété, car elle pensait que cet accord n'était qu'une condition préalable à la réconciliation.

En août 2010, Amol a emménagé dans l'appartement du sous-sol avec Deepa et les enfants. En novembre 2010, Amol a acheté une propriété en son nom propre et la famille a emménagé dans le nouveau domicile. Les parents d'Amol ont rendu visite au couple à l'occasion de la nouvelle année et ils sont restés six mois. Après leur départ, Amol est parti assister au mariage d'un ami en Inde. En décembre 2011, il est retourné au Canada pour célébrer l'anniversaire des jumeaux. Le lendemain, Deepa a reçu un appel téléphonique anonyme l'informant que son mari s'était marié pendant son séjour en Inde. Deepa a fouillé les bagages d'Amol et a trouvé un DVD du mariage. Elle ignorait qu'Amol avait demandé le divorce dans une ville voisine et que l'ordonnance avait été prononcée en avril 2010, sept mois avant son voyage en Inde. Deepa n'avait jamais reçu la demande de divorce et elle ignorait que son mari pouvait obtenir le divorce sans le notifier officiellement par des documents ${ }^{1}$. Elle a demandé des explications à Amol qui a quitté la maison en décembre 2011. Deepa a fait une demande auprès du programme Ontario au travail et a reçu une aide sociale pendant huit mois. En septembre 2012, elle a trouvé un emploi à temps partiel de chauffeuse d'autobus scolaire. Deepa a continué à vivre avec ses enfants dans la maison d'Amol. Après son départ, Amol a appelé plusieurs fois le domicile conjugal, en personne et par l'intermédiaire d'amis, pour exiger que Deepa quitte les lieux.

À partir de 2013, Amol a commencé à ne plus payer les factures d'eau et d'électricité du domicile. L'eau a été coupée et la famille n'avait plus d'eau ni de chauffage pendant de nombreux mois, y compris pendant l'hiver. Deepa et ses enfants étaient contraints de se laver au centre communautaire local. Au début de mai 2015, Amol a arrêté de payer les mensualités hypothécaires et les charges de la maison. La banque a alors engagé une procédure judiciaire pour récupérer la propriété et Deepa et les enfants ont été forcés de quitter la maison.

\section{Résolution}

1. 1. Après enquête, l'avocat de Deepa a pu découvrir ce qui s'était passé. En août 2010, Amol reprenait la vie commune avec Deepa et les enfants. En septembre, il a demandé le divorce auprès de la Cour suprême de justice (1 mois après leur réconciliation) et le divorce a été prononcé en avril 2011. Le tribunal avait constaté l'absence de Deepa et avait prononcé le divorce par défaut. L'avocat a découvert qu'Amol avait rempli un affidavit de signification auprès d'un huissier de justice pour déclarer sous serment qu'il avait personnellement notifié Deepa de la demande de divorce « en lui remettant une copie » (qu'elle n’avait jamais reçue). Deepa n’avait jamais vécu à l’adresse indiquée sur la demande de divorce où l'huissier de justice prétendait lui avoir remis personnellement une copie. L'huissier de justice a été inclus dans la liste des témoins. Le tribunal s'était fondé sur l'authenticité de l'affidavit de signification pour considérer que Deepa avait été dûment notifiée. Le délai de 30 jours pour sa réponse étant expiré, elle a été déclarée en défaut et le tribunal a accordé le divorce. 
Le principal point de désaccord dans cette affaire concerne la date officielle de la séparation entre les parties. Deepa vivait avec Amol et pensait que le mariage était valide jusqu'en décembre 2011, date à laquelle Amol a quitté la maison, bien que l'accord de séparation ait été signé en juin 2009. Ce litige a des conséquences sur le paiement des pensions alimentaires pour les enfants et l'épouse. Amol s'est présenté devant les tribunaux en novembre 2012 en affirmant que Deepa avait monté les enfants contre lui et avait restreint son droit de visite. Il a ensuite demandé la garde et le droit de visite des enfants. Deepa a réfuté l'accusation selon laquelle elle avait restreint le droit de visite et a déclaré qu'Amol n'avait fait aucun effort pour voir ses enfants au cours des onze derniers mois, bien que leur fille soit très malade. Deepa a demandé au tribunal d'annuler la séparation précédente. Elle a demandé la garde exclusive des enfants avec un droit de visite surveillé pour Amol. Elle a demandé que les enfants aient leur propre avocat, une pension alimentaire pour les enfants et les époux et une ordonnance de non-communication contre Amol. 
9.

\section{ÉTUDE DE CAS 8 : CLAUDIA ET HENRY}

\begin{tabular}{|c|c|c|}
\hline Profil & Femme & Homme \\
\hline Nom & Claudia & Henry \\
\hline Âge au moment du mariage & 24 & 46 \\
\hline Âge* & 32 & 54 \\
\hline Pays d'origine & La Dominique & La Dominique \\
\hline Religion & Chrétienne & Chrétienne \\
\hline Éducation & Études secondaires inachevées & Études secondaires \\
\hline Connaissance de l'anglais & Bon niveau & Bon niveau \\
\hline Emploi avant la migration & Sans emploi & Aucune information disponible \\
\hline Emploi & Sans emploi & $\begin{array}{l}\text { Travailleur autonome : nettoyage de } \\
\text { vitres }\end{array}$ \\
\hline Catégorie d'immigration & $\begin{array}{l}\text { Regroupement familial : Henry a } \\
\text { parrainé Claudia dans le cadre du } \\
\text { programme de parrainage des } \\
\text { conjoints. }\end{array}$ & Aucune information disponible \\
\hline Statut d'immigrant* & Résident permanent & Citoyen \\
\hline \multicolumn{3}{|c|}{$\begin{array}{l}\text { Nombre d'années de mariage* : } 8 \\
\text { Enfants* : } \\
\text { - Fille : Faith }(5 \text { ans }) \\
\text { - Fille : Hope ( } 5 \text { ans })\end{array}$} \\
\hline
\end{tabular}

*Au moment de la demande auprès de la Cour de la famille

\section{Parcours avant la migration et l'installation au Canada}

Claudia est arrivée au Canada avec un visa de visiteur en 1999. Elle a rencontré Henry en 2004. Henry était travailleur autonome et vivait au Canada depuis 15 ans. Le couple a commencé à vivre ensemble dans une maison appartenant à Henry. Claudia a dépassé la durée de séjour autorisée pour son visa de visiteur et elle a 
été déportée en 2006. Elle était enceinte des jumeaux à l'époque. Claudia a vécu avec ses filles à la Dominique pendant six années. Henry a parrainé l'immigration de Claudia et de leurs filles au Canada. Elles sont arrivées au Canada en 2012. Henry a dû prouver grâce à des tests ADN qu'il était bien le père des deux filles avant de pouvoir les parrainer. Claudia a acquis un statut de résidence permanente conditionnelle. À son arrivée, la famille s'est installée dans un appartement loué, car Henry avait vendu sa maison.

\section{Violence domestique}

Dès le début de leur relation, Henry a maltraité Claudia financièrement et psychologiquement. Il contrôlait tout. Il ne fournissait pas la nourriture et ne subvenait pas aux dépenses quotidiennes. Il injuriait Claudia et se montrait suspicieux à son égard. Il voulait contrôler toutes les interactions de Claudia avec d'autres personnes. Il gardait l'unique clé de l'appartement et surveillait les allées et venues de tout le monde. Henry était alcoolique. Il était bruyant et agressif quand il avait trop bu.

La situation au sein du ménage s'est détériorée. Outre sa consommation excessive d'alcool, la conduite d'Henry était assimilable à de l'abandon. Il ne donnait pas d'argent à Claudia pour acheter de la nourriture et des vêtements et ne s'occupait pas des enfants. Les filles avaient peur de leur père et disaient qu'il sentait mauvais. Elles ne voulaient pas se trouver en même temps qu'Henry au domicile conjugal. Elle souhaitait s'éloigner de leur père.

Lorsque les filles ont commencé à suivre des cours à l'école, elles ont commencé à avoir des problèmes de comportement (par exemple jeter des objets, faire pipi au lit) et à s'éloigner de leur père. Lors d'un entretien à l'école, Claudia a parlé du comportement d'Henry au professeur de ses deux filles. Henry a été convoqué à l'école et il a eu un accès de colère. Il a commencé à crier après Claudia et à se comporter de manière agressive. Après la réunion, au lieu de retourner au travail, il a suivi Claudia pour s'assurer qu'elle ne quittait pas la maison. Le professeur a appelé la Société de l'aide à l'enfance (SAE) qui est intervenue immédiatement. Elle est restée en contact avec la famille pendant deux ans. Le lendemain, Claudia a reçu la visite d'un ami au domicile conjugal et Henry a commencé à les injurier. Il a menacé Claudia en lui disant qu'il allait lui retirer son statut de résidente permanente. Quelques jours plus tard, en octobre 2012, Claudia a déménagé dans un refuge avec ses filles pour assurer leur sécurité. Elle résidait au Canada depuis moins de six mois.

\section{Résolution}

Claudia a continué à s'occuper de ses enfants après la rupture de vie commune. Elle est la personne qui s'est toujours occupé des enfants. Henry a demandé la garde des enfants et ne voulait pas payer de pension alimentaire : il estimait avoir déjà déboursé une somme importante pour assurer le parrainage de sa famille. L'affaire a été portée devant la Cour de la famille en 2013 et s'est terminée en 2015. Le tribunal a accordé la garde exclusive des enfants à Claudia. Henry a obtenu un droit de visite de jour à la condition qu'il ne consomme pas d'alcool avant et pendant les visites. Le tribunal a rendu une ordonnance de non-communication mutuelle. Claudia a dû retirer sa demande de pension alimentaire. Depuis 2012, la Société d'aide à l'enfance (SAE) est saisie de cette affaire même si elle concerne pas la protection des enfants. Elle apporte un soutien à Claudia et 
aux enfants en leur fournissant des services de counseling, une aide au logement et un accès à des ressources. Claudia continue de recevoir l'aide du gouvernement et subvient à tous les besoins de ses filles. 
ÉTUDE DE CAS 9 : RABIA ET ALI

\begin{tabular}{|c|c|c|}
\hline Profil & Femme & Homme \\
\hline Nom & Rabia & Ali \\
\hline Âge au moment du mariage & 21 & 25 \\
\hline Âge* & 36 & 40 \\
\hline Pays d'origine & Pakistan & Pakistan \\
\hline Religion & Musulman & Musulman \\
\hline Éducation & $\begin{array}{l}\text { Obtention partielle d'un diplôme de } \\
\text { premier cycle en microbiologie de } \\
\text { l'Université de Karachi }\end{array}$ & Premier cycle universitaire \\
\hline Connaissance de l'anglais & Limitée & Limitée \\
\hline Emploi avant la migration & Sans emploi & $\begin{array}{l}\text { Propriétaire d'une concession } \\
\text { automobile }\end{array}$ \\
\hline Emploi & Sans emploi & $\begin{array}{l}\text { Possède et gère une société } \\
\text { concessionnaire automobile }\end{array}$ \\
\hline Catégorie d'immigration & Visa d'entrepreneur & Visa d'entrepreneur \\
\hline Statut d'immigrant* & Résident permanent (PR) & Résident permanent (PR) \\
\hline $\begin{array}{l}\text { Nombre d'années de mariage } \\
\text { Enfants* : } \\
\text { - Fils : Akbar ( } 13 \text { ans }) \\
\text { - Fille : Rashida ( } 8 \text { ans }) \\
\text { - Fille : Rukshana ( } 5 \text { ans }) \\
\text { - Fille : Ala ( } 4 \text { ans }) \\
\text { - Fille : Sultana ( } 4 \text { ans }) \\
\text { - Fille : Afroz ( } 3 \text { mois) }\end{array}$ & & \\
\hline
\end{tabular}

*Au moment de la demande auprès de la Cour de la famille

\section{Parcours avant la migration}


Au début de leur mariage, le couple a déménagé au Moyen-Orient où Ali a créé une concessionnaire automobile. Il a fait déménager sa famille, bien que son fils souffre d'asthme et que le climat du MoyenOrient ne lui convienne pas. Après quatre années de mariage, Rabia et Ali ont immigré au Canada avec leur jeune fils dans le cadre du programme Chefs d'entreprise. Ali apportait plus de 300000 USD, une condition du programme de visa pour le démarrage d'entreprise. Initialement, Rabia avait décidé de ne pas résider au Canada, elle accompagnait simplement son mari pour garder la famille unie. Toutefois, après avoir constaté que le climat du Canada conviendrait beaucoup mieux à son jeune fils, Rabia a estimé que cette immigration pourrait être bénéfique à son fils : celui-ci n’aurait plus besoin de prendre sa dose quotidienne de stéroïdes pour rester en vie à Dubaï.

\section{Installation au Canada}

À leur arrivée au Canada en mai 1999, Rabia et Ali ont acheté au comptant un appartement en copropriété. Ali a ouvert un magasin de meuble tout en continuant à s'occuper de sa concession automobile au MoyenOrient. Deux ans plus tard, le couple a fait une hypothèque pour acheter une deuxième propriété. Cette propriété a été vendue en 2003, mais Rabia n’a reçu aucune part du produit de la vente. Lorsque Rabia et Ali sont devenus résidents permanents (RP) en 2002, Ali a vendu son entreprise de meubles en réalisant un profit. Il a continué à investir à Dubaï. Rabia restait à la maison pour s'occuper de la famille grandissante. Elle était chargée de toutes les tâches domestiques et de l'éducation des enfants. La seule priorité d'Ali, c'était de gagner de l'argent. Cinq ans après son arrivée au Canada, Ali a créé une entreprise d'exportation de voitures, ce qui lui a permis d'acheter et de transférer des voitures au Moyen-Orient.

\section{Violence domestique}

Tout au long de leur mariage, Ali a maltraité verbalement et physiquement Rabia en présence des enfants. Il a exhibé ses relations avec d'autres femmes sous les yeux de sa femme et a exigé d'elle des rapports sexuels à sa seule convenance. Il contrôlait l'argent de Rabia, surveillait leurs comptes par voie électronique et ne lui fournissait que l'argent strictement nécessaire. Il refusait toutes les demandes d'argent supplémentaire visant à financer les activités extrascolaires de leurs enfants. Elle n'avait pas le droit de quitter la maison, sauf en cas de nécessité absolue et n'était pas autorisée à contacter sa famille vivant au Pakistan. Durant leur mariage, Ali a forcé Rabia et ses enfants à l'accompagner dans ses voyages d’affaires prolongés au Moyen-Orient (jusqu’à six mois) malgré l'état de santé fragile de leur fils. Rabia était obligé de retirer ses enfants de l'école pour pouvoir accompagner son mari. Au cours de ces voyages, Rabia et ses enfants restaient enfermés à la maison tandis qu'Ali traitait ses affaires et rentrait tard dans la soirée. De retour au Canada, Rabia réinscrivait les enfants à l'école, mais ils ne parvenaient pas à rattraper leur retard scolaire.

Ali criait et frappait souvent ses enfants qui avaient peur de leur père. Ali menaçait régulièrement Rabia de prendre les enfants au milieu de la nuit pour les mettre sur un vol pour le Pakistan et que celle-ci ne pourrait plus les revoir. En février 2009, Rabia a décidé de contacter la police pour dénoncer les sévices qu'elle avait subis toutes ces années. Deux de ses filles, âgées de six et neuf ans, ont révélé qu’elles avaient été agressées sexuellement 
par Ali. Son fils a déclaré qu'il avait été maltraité physiquement par Ali qui le filmait sous la douche. Dans un premier temps, l'Unité spéciale des victimes (SVU) était sceptique face à ces allégations, car elle estimait que les enfants étaient manipulés. Toutefois, la Société d'aide à l'enfance (SAE) a décidé en décembre d'inscrire Ali sur le Registre de l'enfance maltraitée. Ali a été accusé de deux chefs d'accusation d'agression et d'un chef d'accusation d'agression sexuelle sur Rabia. Il a été libéré sous caution et la Cour de la famille lui a ordonné de n'avoir aucun contact direct ou indirect avec la mère ou les enfants. Il n'a pas été autorisé à quitter le pays. En mai 2009, il s'est présenté devant la cour et a obtenu une modification de sa libération conditionnelle qui lui a permis de récupérer son passeport et de pouvoir voyager à des fins professionnelles. Quelques mois après l'ordonnance, le frère et la sœur d'Ali ont commencé à harceler Rabia en lui téléphonant ou en lui rendant visite pour lui demander de régler cette affaire sans passer par le tribunal. Son beau-frère lui laissait des messages vocaux pour la menacer de lui enlever ses enfants si elle refusait de lui parler. Il invoquait aussi la colère de Dieu qui la punirait. Un jour, son beau-frère est venu frapper agressivement à la porte de son appartement et a effrayé les enfants. Elle a alors décidé d’appeler le 911 . La police est venue la voir pour l'informer que son beau-frère avait reçu un avertissement lui demandant de ne plus la contacter. Elle a demandé à la Cour de la famille une ordonnance d'éloignement pour empêcher son mari ou toute personne agissant en son nom de la contacter.

\section{Résolution}

Ali a plaidé coupable pour les charges d’agression contre Rabia. Par contre, les accusations d'agression sexuelle ont été abandonnées. Il a été condamné à une probation de douze mois avec interdiction de tout communication avec Rabia et les enfants. En outre, il a dû participer à des séances de gestion de la colère. En août 2011, il a reçu l'ordre de remettre son passeport à son avocat et de fournir une déclaration de sa situation financière complète. Il n’a exécuté aucun de ces ordres. 
ÉTUDE DE CAS 10 : ANJANA ET MARC

\begin{tabular}{|c|c|c|}
\hline Profil & Femme & Homme \\
\hline Nom & Anjana & Marc \\
\hline Âge au moment du mariage & 33 & 34 \\
\hline Âge* & 51 & 52 \\
\hline Pays d'origine & Guyana & Guyana \\
\hline Religion & Chrétienne & Chrétienne \\
\hline Éducation & Études secondaires & Études secondaires \\
\hline Connaissance de l'anglais & Capable de communiquer en anglais & Bon niveau \\
\hline Emploi avant la migration & Sans emploi & Aucune information disponible \\
\hline Emploi & Opérateur industriel & Sans emploi \\
\hline Catégorie d'immigration & $\begin{array}{l}\text { Regroupement familial : Marc a } \\
\text { parrainé Anjana (parrainage de } \\
\text { conjoint) }\end{array}$ & $\begin{array}{l}\text { Regroupement familial. Marc a été } \\
\text { parrainé par sa mère avant le mariage. }\end{array}$ \\
\hline Statut d'immigrant* & Citoyen & Citoyen \\
\hline \multicolumn{3}{|c|}{$\begin{array}{l}\text { Nombre d'années de mariage* : } 18 \\
\text { Enfants* : }^{*} \\
\text { - Fille : Abi (22 ans }) \\
\text { - Fille : Susan ( } 20 \text { ans }) \\
\text { - Fille : Christina (14 ans) } \\
\text { - Fils : Sean (12 ans) }\end{array}$} \\
\hline
\end{tabular}

*Au moment de la demande auprès de la Cour de la famille

\section{Parcours avant la migration}

Anjana et Marc se sont mariés au Guyana en août 1992. Il s'agissait d'un premier mariage pour les deux. La mère de Mark qui vivait au Canada avait parrainé son fils pour qu'il puisse immigrer au Canada en 1989. Mark et Anjana avaient eu un enfant en 1988 et un second en 1992. Après leur mariage en 1992, Mark a parrainé 
Anjana pour qu'elle immigre au Canada. Elle est arrivée avec ses deux filles ( 4 ans et 2,5 ans). Deux autres enfants sont nés après l'immigration : une fille en 1996 et un fils en 1998.

\section{Installation au Canada}

Le couple a acheté une maison dans une grande ville canadienne. Mark était alors au chômage et avait peu de facilité de crédit et la propriété a été achetée au nom d’Anjana. Pendant 13 ans, elle a payé toutes les mensualités du prêt hypothécaire et a occupé trois emplois pour pouvoir payer les dépenses du ménage. Mark n’a jamais eu d'emploi stable et ne soutenait pas financièrement Anjana et sa famille. Anjana achetait toute la nourriture et les vêtements des enfants avec son argent. À plusieurs reprises, Anjana a dû emprunter de l'argent à des amis ou à sa famille pour pallier le manque à gagner et ne pas perdre le domicile conjugal. Anjana a fait face à de multiples problèmes de santé (hypertension, polyarthrite rhumatoïde et syndrome du tunnel carpien), mais s'est toujours forcée à travailler.

\section{Violence domestique}

Pendant les dix-huit années de leur mariage, Mark a maltraité physiquement et verbalement Anjana et leurs enfants. Il contrôlait tous les faits et gestes de son épouse : il écoutait ses appels téléphoniques, la suivait au travail et consultait ses messages électroniques. La nuit, Mark fouillait dans le portefeuille d'Anjana et vérifiait son compte bancaire et ses transactions. Pendant toute cette période, la police a été appelée à de nombreuses reprises : Anjana et ses enfants ont appelé la police vingt-et-une fois pour des faits de violence et Mark a appelé la police à douze reprises pour se plaindre de violences verbales. Peu après l'arrivée d’Anjana au Canada, Mark a agressé sa femme en la frappant à la tête. La police a été appelée et Mark a été inculpé. Anjana est restée dans un refuge avec ses filles. Mark a été condamné et mis en liberté conditionnelle. Le couple a vécu séparément, mais s'est réconcilié un an et demi plus tard. Mark a aussi maltraité ses enfants. En décembre 2008, Anjana était au travail lorsque Mark s'est mis en colère contre sa deuxième fille, alors âgée de dix-sept ans et l'a agressée physiquement. Il l'a poursuivie dans toute la maison, l’a frappée et lui a lancé un rouleau à pâtisserie. Elle s'est enfuie en courant de la maison, mais Mark l'a rattrapée, a saisi son poignet et a commencé à la frapper. Un voisin, témoin de l'agression, a appelé la police. Mark a été arrêté et inculpé. Il a été mis une nouvelle fois en probation. Mark avait déjà été condamné pour agression de l'enfant d'un voisin et mis à l'épreuve pour cette accusation. En septembre 2010, trois ans après cet incident, Mark a été accusé de plusieurs délits : agression, agression armée, possession d'une arme et «préjudices ». Ces accusations concernaient Anjana et leurs deux enfants. Mark a été libéré et assujetti à de strictes conditions de mise en liberté sous caution. À cette époque, le couple s'était séparé et deux ans plus tard, Mark a été reconnu coupable de voies de fait et de non-respect des conditions de mise en liberté sous caution. Il a été condamné à une période de mise à l'épreuve d'un an avec interdiction de contact avec Anjana et ses enfants. À la fin de sa période de probation, il est retourné de force au domicile conjugal pour y vivre séparément d'Anjana et de ses enfants. Il a pris possession de la chambre principale, du garage et du sous-sol. Anjana ne savait pas quoi faire.

D’autres agressions ont eu lieu, notamment une agression sur une autre fille et Mark a fait l'objet de nouvelles 
accusations. Mark a reçu l'ordre de quitter le domicile conjugal et de récupérer ses affaires sous surveillance. Il a passé outre cette injonction pour entrer dans la maison (en présence de son fils) et emporter de nombreux objets, dont des effets de famille précieux. En novembre 2014, Mark et son fils (alors âgé de presque 16 ans) ont eu une altercation et Mark a frappé son fils. Anjana a eu connaissance de cet incident trois semaines plus tard, lorsque son fils lui en a parlé.

Quand il habitait au domicile conjugal, Mark n'a jamais contribué financièrement à son entretien : il n'a pas payé les taxes foncières pendant trois (3) ans et Anjana a reçu une facture d'impayés de 10000 dollars. Les enfants ont été obligés de demander à leur grand-mère paternelle de prêter de l'argent à Anjuna, car Anjana n'avait pas les moyens de payer la facture. La grand-mère leur a prêté de l'argent. Mark a promis de payer la moitié de la somme, mais il ne l'a jamais fait.

Mark a tenté de détourner les allocations familiales versées à Anjana en demandant à l'administration fiscale de mettre ces chèques à son nom. La santé d'Anjana a décliné en réaction au stress et à la violence et elle a commencé à avoir des crises de panique.

\section{Résolution}

Mark est censé payer une pension alimentaire, mais n’a pas déclaré avec exactitude ses revenus et ses biens (voiture de collection) obtenus par des activités illégales. La date de leur séparation est contestée. Le domicile conjugal a été vendu et Anjana a reçu une part égale du partage des biens, ainsi que les arriérés de pension alimentaire des enfants (séparation de 2010 à 2015). Mark a également obtenu une part égale du partage des biens. Le tribunal a interdit à Mark de communiquer avec Anjana, sauf par l'intermédiaire d'un avocat. Mark doit payer une pension alimentaire pour l'enfant le plus jeune sur la base d'un salaire minimum. Le divorce a été officialisé en 2016. 


\section{ÉTUDE DE CAS 11 : SHOVA ET ANANT}

\begin{tabular}{|l|l|l|}
\hline Profil & Femme & Homme \\
\hline Nom & Shova & Anant \\
\hline Âge au moment du mariage & 23 & 25 \\
\hline Âge* & 38 & 40 \\
\hline Pays d'origine & Inde & Inde \\
\hline Religion & Hindou & Jain \\
\hline Éducation & Premier cycle universitaire & Premier cycle universitaire \\
\hline Connaissance de l'anglais & Bon niveau & Bon niveau \\
\hline Emploi avant la migration & Architecte d'intérieur & $\begin{array}{l}\text { Possède une entreprise d'importation } \\
\text { et d'exportation en Inde }\end{array}$ \\
\hline Emploi & Commerce de détail & Propriétaire d'une société \\
\hline Catégorie d'immigration & $\begin{array}{l}\text { Permis de travail temporaire (2007) } \\
\text { Résidence permanente au titre du } \\
\text { programme de visa pour démarrage } \\
\text { d'entreprise en 2009 }\end{array}$ & $\begin{array}{l}\text { Permis de travail temporaire (2007) } \\
\text { Résidence permanente au titre du } \\
\text { d'entreprise en 2009 }\end{array}$ \\
\hline $\begin{array}{l}\text { Statut d'immigrant* } \\
\text { Enfants* }:\end{array}$ & Résident permanent (2009) & Résident permanent (2009) \\
\hline 15 & ans, citoyen canadien) \\
\hline
\end{tabular}

* Au moment du dépôt de la demande auprès de la Cour de la famille

\section{Parcours avant la migration}

En Inde, Shova travaillait comme architecte d'intérieur et hôtesse dans un grand aéroport international. Anant possédait une entreprise d'import-export en Inde. Il a développé cette entreprise en immigrant au Canada. Shova a travaillé pour l'entreprise de son mari : elle exerçait des fonctions dans la vente et le marketing 
en Inde et au Canada. Après leur mariage, Shova et Anant ont vécu dans la famille élargie d'Anant (16 personnes). Leur mariage était assorti de deux conditions : Shova devait renoncer à manger de la viande, des oignons et de l'ail (interdit par la religion) et elle devait rompre tous les liens avec sa famille. Anant était un pratiquant convaincu de la religion jaïne. Par respect pour les croyances de son époux, Shova a renoncé à manger de la viande à partir de leur mariage. Mais Anant a toujours soupçonné sa femme de ne pas respecter cet engagement.

Dès le début, le mariage était instable, car Shova n'était pas jaïne et elle venait d'une communauté qui mangeait de la viande, des oignons et de l'ail. Un après leur mariage, Anant a agressé une première fois Shova. Puis Anant et sa famille ont voulu annuler le mariage afin qu'il puisse épouser quelqu'un de la communauté jaïne. Ils ont demandé à Shova de quitter le domicile conjugal et de retourner chez ses parents. Après discussion entre les familles élargies, le couple s'est réconcilié. Toutefois, les mauvais traitements physiques, psychologiques, verbaux et sexuels se sont poursuivis.

Peu après, Shova est tombée enceinte. Elle a été admise deux fois à l'hôpital durant sa grossesse. La première fois, Anant lui avait donné un coup de pied et l'avait forcé à dormir à même le sol en marbre froid. La seconde fois, Anant l'avait poussé dans une baignoire, en l'accusant de ne pas l'avoir nettoyé : Shova souffrait de contusions abdominales. Le cousin d'Anant l'a conduite à l'hôpital où elle est restée pendant cinq jours dans l'unité de soins intensifs pour surveiller l'état de santé du foetus. Shova a informé son médecin de l'incident et Anant a reçu l'ordre de ne pas se rendre à l'hôpital. Lorsque la famille de Shova a demandé des explications à Anant à propos de son comportement, il s'est montré agressif et irrespectueux envers eux.

Shova a donné naissance à son enfant en 2002. L'hôpital a alors décidé de prendre des mesures de sécurité pour éviter qu'Anant ne prenne le bébé. Mais cette menace ne s'est pas réalisée. Durant les premières années de la vie de son fils, Shova a voyagé fréquemment aux États-Unis pour aider Anant à développer son entreprise. Cinq ans plus tard, Shova et Anant ont immigré au Canada avec un visa de travail temporaire en 2007 et un titre de résident permanent dans le cadre du programme de visa pour démarrage d'entreprise en 2009. Ils ont immédiatement ouvert une filiale au Canada de leur entreprise d'import-export indienne. Shova a repris ses activités de vente et de marketing dans la nouvelle structure.

\section{Installation au Canada}

Shova a commencé à travailler à temps partiel pour une grande chaine de magasins de bricolage tout en contribuant à l'entreprise familiale qui ne fournissait pas encore de revenus stables. Elle devait aussi s'occuper de toutes les tâches domestiques et de l'éducation des enfants. À l'époque, le couple ne possédait qu'une seule voiture et Shova devait marcher une demi-heure dans la nuit froide, quand Anant oubliait de venir la chercher. Elle communiquait rarement avec sa famille en Inde, car Anant limitait ses contacts avec eux. Elle n'avait pas non plus d'amis, car Anant les faisait fuir par son contrôle de tous ses faits et gestes de sa femme. Un an après son arrivée au Canada, Shova a donné naissance à son deuxième fils. L’accouchement difficile a nécessité une césarienne. Anant n’a apporté aucune aide et ne s'est pas intéressé à la santé de sa femme et du bébé. Toute l'attention d’Anant était consacrée à son entreprise. En 2009, le couple a loué un terrain pour développer 
ses activités. Tous les salaires perçus par Shova dans son emploi de vendeuse ont été absorbés par l'entreprise familiale. L'année suivante, le couple a acheté une maison.

\section{Violence domestique}

Dès le début de leur mariage, Anant s'est montré violent avec son épouse. Il l'a accusé à tort d'avoir une liaison extraconjugale. Shova n’a jamais parlé de ces mauvais traitements, car Anant était respecté dans la communauté. Elle souhaitait préserver sa réputation. La situation a empiré lorsque Shova a été promu à un poste de cadre à plein temps en 2012. Après en avoir discuté avec Anant, elle a accepté les nouvelles responsabilités associées à cette promotion. Le contrôle exercé par Anant s'est alors intensifié. Il l'appelait ou passait la voir plusieurs fois par jour à son travail, ce qui perturbait son activité et énervait ses collègues. Il lui imposait un contrôle total de ses finances en surveillant toutes ses dépenses, ses investissements et ses cartes de crédit. La pression exercée par la surveillance d’Anant, son emploi du temps surchargé (salarié et domestique) et le soin apportés aux enfants est devenue trop forte: Shova a été hospitalisée pour une paralysie temporaire due au stress.

En 2013, Shova et Anant ont vendu leur première maison pour acheter une nouvelle maison. Les enfants ont été scolarisés dans un nouvel établissement. Peu après le déménagement, Anant a violemment battu leur fils aîné au point de lui laisser des marques sur le visage. Quelques jours plus tard, leur fils cadet a raconté à l'école ce qui s'était passé. L’école a appelé Shova et lui a dit qu'il était de sa responsabilité de protéger ses enfants. Après une visite de la SAE au domicile familial, Anant a cessé de maltraiter physiquement ses enfants tout en continuant à les agresser verbalement. Sur recommandation de la SAE, les garçons ont bénéficié d'un soutien psychologique.

En 2014, Anant a agressé physiquement Shova si violemment qu'elle avait marchait difficilement après deux jours d'arrêt de travail. La dispute avait éclaté, car Anant voulait que sa femme porte une robe avec un décolleté plongeant pour assister à un événement professionnel. Lorsque celle-ci a refusé, il l'a frappée et jetée contre des étagères. Il a déchiré ses vêtements jusqu’à ce qu'elle accepte de porter ce qu'il voulait. Bien qu'elle ait été blessée au cours de cette altercation, elle a décidé de ne pas aller chez son médecin et de retourner à son travail après un congé de deux jours. Après avoir tenté de dissimuler sa douleur à ses collègues, sa conduite a éveillé les soupçons d'un collègue de travail, qui a signalé le mauvais traitement au bureau des ressources humaines de l'entreprise. On lui a conseillé d’appeler une ligne d'assistance téléphonique pour obtenir de l'aide et des conseils. En novembre 2014, Anant a apposé frauduleusement et à l'insu de Shova le nom de celle-ci sur les documents de constitution d'une nouvelle société. Au cours des années suivantes, il a continué à utiliser illégalement le nom de Shova dans un but lucratif.

En février 2015, le couple a eu une violente dispute et Anant a chassé Shova de la maison. À cette occasion, Anant a menacé Shova de la tuer si jamais elle revenait. Encore sous le choc, elle est allée travailler et a appelé Anant toute la journée, car elle était préoccupée pour la sécurité des enfants. Anant a ignoré ses appels téléphoniques. Elle a trouvé un logement provisoire chez une amie, car elle ne se sentait pas en sécurité chez elle. Dans les semaines qui ont suivi, Anant a essayé de la faire revenir à la maison. En avril 2015, il a menacé 
de se suicider par overdose et lui en fait porter la responsabilité. Ce soir-là, elle a rendu visite à ses enfants, mais Anant s'est jeté sur elle. Il a brandi un couteau et a menacé de la tuer et de se tuer. Effrayée, elle a appelé le 911. La police est arrivée et Anant a été transféré à l'hôpital, mais aucune charge n’a été retenue contre lui. Les enfants étaient traumatisés par la scène à laquelle ils avaient assisté et Shova a décidé de les emmener avec elle pour une nuit. Le lendemain, Anant est sorti de l'hôpital et il est allé chercher les enfants à l'école. Les enfants ont commencé à communiquer en cachette avec leur mère.

Au début de la séparation, Anant avait vidé le compte bancaire commun et interdit à Shova de revenir. L'absence de Shova au sein du foyer conjugal se faisait sentir. Le garçon le plus âgé, qui avait douze ans à l'époque, assumait souvent le rôle de parent de substitution pour son frère cadet; il devait lui administrer ses médicaments en cas de besoin.

Shova et Anant ont alors négocié un accord informel sur le rôle parental de chacun. Shovan, qui vivait à l'extérieur, devait se rendre au foyer conjugal pour réveiller les garçons et les aider à préparer leur journée. Elle devait s'occuper des affaires scolaires, préparer les déjeuners, faire la lessive, nettoyer la maison et déposer les enfants à l'école. Elle pouvait leur rendre visite certains soirs, mais elle ne pouvait pas voir les enfants chez elle ou les garder les fins de semaine.

\section{Résolution}

Anant a reproché à Shova d’avoir abandonné ses enfants. Shova a sollicité l'aide d'un avocat et demandé la garde conjointe des enfants, y compris une pension alimentaire pour les enfants et pour le conjoint. Shova a demandé au Bureau de l'avocat des enfants d'intervenir dans cette affaire pour défendre les intérêts des enfants. Sa demande a été acceptée. En 2016, la cour a accordé la garde conjointe aux deux parties avec le domicile du père comme résidence principale. Le problème des biens a été résolu par le biais d'un règlement financier. Anant et Shova vivent actuellement avec de nouveaux partenaires. 


\section{ÉTUDE DE CAS 12 : BINA ET UDEEP}

\begin{tabular}{|c|c|c|}
\hline Profil & Femme & Homme \\
\hline Nom & Bina & Udeep \\
\hline Âge au moment du mariage & 23 & Aucune information disponible \\
\hline Âge* & 55 & Aucune information disponible \\
\hline Pays d'origine & Inde & Inde \\
\hline Religion & Chrétienne & Hindou \\
\hline Éducation & Diplôme d'infirmière & Premier cycle universitaire \\
\hline Connaissance de l'anglais & Bon niveau & Bon niveau \\
\hline Emploi avant la migration & Infirmière & Aucune information disponible \\
\hline Emploi & Emploi dans un salon funéraire & Aucune information disponible \\
\hline Catégorie d'immigration & $\begin{array}{l}\text { Bina est entrée au Canada avec un } \\
\text { visa de visiteur et a demandé le } \\
\text { statut de réfugié. }\end{array}$ & Sans objet (pas d'immigration) \\
\hline Statut d'immigrant* & $\begin{array}{l}\text { Demande de titre de séjour pour des } \\
\text { raisons humanitaires }\end{array}$ & Aucune information disponible \\
\hline \multicolumn{3}{|c|}{$\begin{array}{l}\text { Nombre d'années de mariage* : } 29 \text { (1986-2015, séparation en 2010) } \\
\text { Enfants* : } \\
\text { - Fils : Vijay (né en mai 1987, handicap à la suite d'un accident de moto en 2009) } \\
\text { - Fils : Raju (15 ans), trouble déficitaire de l'attention (TDAH), syndrome de stress post-traumatique et } \\
\text { dépendance aux jeux vidéo) }\end{array}$} \\
\hline
\end{tabular}

*Au moment de la demande auprès de la Cour de la famille

\section{Parcours avant la migration}

Bina et Udeep se sont mariés par amour, mais leur mariage interreligieux n’a pas été accepté par leur famille. Lors du mariage, la famille de Bina a rompu tout lien avec elle. Bina a vécu par intermittence avec Udeep à 
Bahreïn pendant treize ans (de 1997 à 2010). Son fils aîné ne l’a pas accompagnée à Bahreïn. Il est resté en Inde et le fils cadet est né à Bahreïn.

À Bahreïn, Bina était maltraité par son mari qui lui faisait subir des violences psychologiques, verbales, physiques et financières. Alcoolique, Udeep frappait Bina au visage et sur le corps. Un jour, il lui a coincé la main dans la porte d'un placard. Bina a également subi de multiples viols conjugaux. En outre, son exmari injuriait et frappait leur plus jeune enfant Raj. Bina n’a pas signalé ces mauvais traitements aux autorités indiennes, car son mari avait menacé de la tuer si elle en parlait. Elle n'a pas non plus signalé les violences aux autorités de Bahreïn, car elle vivait dans ce pays avec un visa de travail et elle craignait que son visa soit annulé et que sa famille soit expulsée en Inde. Bina a appelé une seule fois la police en Inde et a été violemment battue par Udeep par la suite.

\section{Installation au Canada}

Après des années de mauvais traitements, Bina a quitté Bahreïn le 25 septembre 2010 pour s'enfuir au Canada où elle est entrée avec un visa de visiteur. Elle était accompagnée de son fils Raju, qui avait alors neuf ans. Son fils aîné était resté en Inde. Bina n’avait aucune famille ou ami au Canada. Un mois après son arrivée, elle a sollicité le statut de réfugié pour elle et son fils. Deux ans plus tard, sa demande d'asile a été rejetée, car elle ne pouvait apporter aucune preuve des violences familiales qu'elle avait subies.

En outre, sa naissance en Inde, un pays figurant sur la liste des pays sûrs établie par la Commission canadienne de l'immigration et du statut de réfugié, ne jouait pas en sa faveur. Elle a tenté de faire appel de cette décision, mais son recours a été rejeté par la Cour fédérale. Un an et demi plus tard, Bina a sollicité un titre de résidence permanente pour des raisons humanitaires $(\mathrm{H} \& \mathrm{C})$. Elle a demandé à la Cour de prendre en considération les difficultés inhabituelles, injustifiées et disproportionnées auxquelles elle serait confrontée si elle devait retourner en Inde. Sa demande a été rejetée. Elle était titulaire d'un permis de travail et pouvait travailler au Canada. En 2015, elle a déposé une deuxième demande de résidence permanente pour raisons humanitaires qui a été aussi rejetée, car elle n’avait pas travaillé à plein temps au cours de ses cinq années de séjour au Canada. Un arrêté d'expulsion a alors été prononcé contre Bina et Raju. Celle-ci a fait appel. L'arrêté d'expulsion a été suspendu jusqu’à ce que l'appel soit examiné. Raju était terrifié à l'idée d'être expulsé du Canada. Il a prévenu Bina qu'il la quitterait si cela devait se produire.

\section{Violence domestique}

Durant son séjour au Canada, Bina a échappé aux violences conjugales de son mari, mais a souffert d'un stress constant lié à la crainte d'être expulsée et au traumatisme des sévices endurés en Inde et à Bahreïn. Son mari, Udeep, avait déclaré à la mère de Bina (qui vivait en Inde) qu'il attendait le retour de Bina. Il avait menacé de s'en prendre à Bina si jamais elle revenait en Inde ou a Bahreïn. Bina vivait donc dans une peur constante de mourir si elle était expulsée et forcée de rentrer chez elle en Inde. Elle a tenté de se suicider à plusieurs reprises à cause des souvenirs douloureux des sévices imposés par son ex-mari.

Bina a aussi subi des violences physiques de la part de son fils. Confronté au traumatisme des mauvais 
traitements passés et à la peur d'être expulsé, son fils avait des problèmes à l'école. Bina rencontrait des difficultés dans son rôle de mère à cause des troubles du déficit de l'attention avec hyperactivité (TDAH), les troubles du stress et la dépendance au jeu de Raju. En juillet 2015, Bina a commencé à travailler comme chauffeur d'autobus pour accroitre ses chances d'obtenir un titre de résidence permanente pour raisons humanitaires. Elle a également fait du bénévolat au niveau local. Elle vivait dans une municipalité, mais travaillait comme chauffeur d'autobus dans une autre municipalité. Il s'agissait d'un travail par roulement fractionné visant à assurer un service de dix-sept heures par jour. En raison de ses horaires de travail, son fils n'était plus surveillé et sa dépendance au jeu s'est aggravée. Il a commencé à voler des cartes de crédit de sa mère pour financer son addiction. Lorsque Bina lui demandait des explications, il devenait violent et menaçait de la tuer. En avril 2016, Raju a agressé Bina. Il l’a poussée et frappée. Bina a appelé le 911. Raju a été inculpé et libéré sous caution. Il a été renvoyé chez lui avec interdiction de sortir à certaines heures de la journée et la Société d'aide à l'enfance a été saisie.

En mai 2016, il a contrevenu aux conditions de sa libération sous caution et n'est pas rentré chez lui. Bina a appelé la police pour signaler sa disparition. Il a été retrouvé quatre jours plus tard, inculpé pour violation des conditions de mise en liberté sous caution et traduit en justice. Bina ne s'est pas présentée au tribunal pour fournir la caution et la SAE a donc pris en charge Raju. Un policier avait conseillé à Bina de laisser la SAE assumer la responsabilité des soins et des évaluations de l'enfant afin de trouver un soutien approprié pour lui. Raju a alors été placé sous la responsabilité de la SAE et placé dans un foyer d'accueil. Raju (aujourd'hui âgé de 16 ans) a alors déclaré à son avocat qu'il ne souhaitait pas retourner sous la garde de sa mère et qu'il voulait devenir un pupille de la Couronne. Bina a été contrainte d'accepter la volonté de Raju, qui est devenu pupille de la Couronne en octobre 2017. Bina a obtenu un droit de visite à la seule discrétion de l'enfant.

Bina a fait appel à de multiples ressources au Canada. Elle a d'abord eu recours à une clinique d'aide juridique à but non lucratif pour l'aider à faire ses demandes de titre de résidence et de statut de réfugié. Elle a aussi bénéficié d'un service de counseling communautaire pour l'aider à surmonter le traumatisme des mauvais traitements qu'elle avait subis avant de venir au Canada.

Elle a suivi des cours d'anglais langue seconde (ALS) et d'éducation parentale pour l'aider à gérer sa relation avec son fils.

\section{Résolution}

En octobre 2017, Raju est devenu pupille de la Couronne et la SAE a embauché un avocat pour aider Raju à faire sa demande d'immigration. Depuis lors, il n'a manifesté aucune volonté de poursuivre sa relation avec sa mère. Bina a déposé séparément une demande pour obtenir une résidence permanente pour raisons humanitaires en décembre 2017. Cette demande n'a pas été examinée et l'Agence des services frontaliers du Canada a expulsé Bina en Inde le 21 janvier 2018. 


\section{ÉTUDE DE CAS 13 : ELHAM ET DAWOOD}

\begin{tabular}{|c|c|c|}
\hline Profil & Femme & Homme \\
\hline Nom & Elham & Dawood \\
\hline Âge au moment du mariage & 20 & 41 \\
\hline Âge* & 35 & 55 \\
\hline Pays d'origine & Aucune information disponible & Aucune information disponible \\
\hline Religion & Musulman & Musulman \\
\hline Éducation & $\begin{array}{l}\text { Enseignement secondaire } \\
\text { (Allemagne) }\end{array}$ & Baccalauréat en génie mécanique \\
\hline Connaissance de l'anglais & Bon niveau & Bon niveau \\
\hline Emploi avant la migration & Sans emploi & Pays du moyen \\
\hline Emploi & Sans emploi & Employé \\
\hline Catégorie d'immigration & $\begin{array}{l}\text { Regroupement familial : Dawood a } \\
\text { parrainé Elham dans le cadre du } \\
\text { programme de parrainage des } \\
\text { conjoints (1995) }\end{array}$ & $\begin{array}{l}\text { Immigration avec un visa étudiant } \\
\text { (en 1980). }\end{array}$ \\
\hline Statut d'immigrant* & Citoyen & Citoyen \\
\hline \multicolumn{3}{|c|}{$\begin{array}{l}\text { Nombre d'années de mariage* : } 13 \\
\text { Enfants* : }^{*}\end{array}$} \\
\hline
\end{tabular}

* Au moment de la demande auprès de la Cour de la famille

\section{Parcours avant la migration}

Avant son mariage, Dawood vivait au Canada depuis quinze ans, où il travaillait comme ingénieur. Il vient d'une famille puissante et influente entretenant des liens avec les services secrets de son pays d'origine. Dawood a parrainé Elham pour que celle-ci puisse immigrer au Canada en 1995, un an après son mariage. Il existe un 
écart d'âge important entre Elham et son conjoint ; elle est de vingt ans plus jeune que lui. Elham a affirmé que Dawood souhaitait se marier avec une femme plus jeune que lui pour pouvoir la contrôler et l'entrainer.

\section{Installation au Canada}

À son arrivée au Canada, Elham a souhaité reprendre ses études pour terminer sa formation de dentiste. Dawood ne l'a pas encouragée à poursuivre dans cette voie. Il lui a dit qu'il voulait qu'elle s'occupe de la maison et fonde une famille. Elham était responsable de tous les aspects de l'éducation des enfants, notamment les devoirs scolaires, les activités parascolaires et les rendez-vous médicaux. Elham avait une sœur qui vivait au Canada, mais le reste de sa famille était resté dans son pays d’origine.

\section{Violence domestique}

Peu de temps après son mariage, Elham a découvert que Dawood entretenait une relation amoureuse durable avec une Canadienne. En fait, Dawood avait invité cette femme à la lune de miel d'Elham et de Dawood, apparemment comme photographe et vidéographe. Cette relation s'est poursuivie tout au long de son mariage avec Elham. Cette femme possédait la clé des trois domiciles conjugaux du couple. Elle était également copropriétaire du premier domicile conjugal d'Elham et de Dawood. Elham était censé se satisfaire de cet arrangement. Elle était isolée à la maison et son époux contrôlait ses fréquentations. Elle ne disposait pas de la clé de la maison et ne pouvait pas aller et venir comme bon lui semblait.

Dès le début de leur mariage, Elham a été victime des mauvais traitements de Dawood. Dawood buvait beaucoup. Il criait, l'insultait, la frappait et la poussait violemment. Il menaçait de la tuer et déclarait que « chaque minute passée en prison en vaudrait la peine si elle était morte ». Il demandait à ses enfants de dire à leur mère qu'ils la détestaient. Les enfants étaient témoins de ses violences et suppliaient leur père de cesser ses mauvais traitements. Elham avait envisagé de contacter la police, mais elle avait renoncé, car son mari lui avait dit qu'il enverrait les enfants hors du Canada et qu'elle ne les reverrait jamais. Dawood était en possession des passeports des enfants, sa famille occupait des postes importants et il travaillait dans le secteur du transport aérien : la menace était donc bien réelle.

Dawood interrogeait sans cesse ses enfants pour savoir ce que faisait Elham pendant la journée et avec qui elle parlait. Les enfants étaient réticents à fournir ces informations sur leur mère. Dawood leur retirait alors leurs jouets et leurs jeux en guise de punition. Il se mettait en colère et réprimandait les enfants. Il se montrait excessivement agressif avec eux, leur reprochant de ne pas être à la hauteur de ses attentes, à la fois dans le sport et à l'école. Il se comportait de manière incohérente : il leur apportait des cadeaux à d'autres occasions. Cette imprévisibilité inspirait aux enfants de la crainte à son égard.

Un jour, Elham a parlé de ces mauvais traitements à son médecin de famille. Celui-ci a commencé à la soigner pour les symptômes qu'elle présentait. Depuis des années, elle prenait des médicaments pour supporter le stress psychologique. Selon les propres dires d'Elham, celle-ci était devenue plus avisée, plus forte et moins tolérante à l'égard de ces mauvais traitements. Malheureusement, Dawood réagissait négativement à la résilience grandissante de son épouse et la violence ne faisait qu'augmenter. 
Il ordonnait à ses enfants de ne plus l'appeler «maman ». Il la traitait comme une servante. Elham n'avait pas d'argent et Dawood ne lui fournissait aucun soutien financier. Elham a alors commencé à travailler à temps partiel comme assistante dans une agence immobilière lorsque ses enfants étaient à l'école. Elham avait obtenu une licence d'agent immobilier, mais Dawood ne lui avait pas permis de le renouveler. Il ne voulait pas qu'elle travaille. Il lui alors demandé de lui remettre directement son salaire. Dawood avait accumulé une dette importante de 600000 dollars à la suite de décisions financières risquées et de fraudes, notamment en demandant trente-six cartes de crédit au nom d'Elham. Dès le début de leur mariage, Dawood avait demandé à Elham de signer un accord postnuptial qui stipulait qu'il aurait la garde des enfants et qu'elle devrait lui verser une certaine somme d'argent en cas de séparation. Elham avait toujours refusé de signer cet accord. Malgré cette situation financière délicate, Dawood conduisait des voitures de luxe et laissait à Elham une vieille voiture qui devait être réparée.

En mars 2008, la famille d'Elham a embarqué pour une croisière d'une semaine en compagnie d'une autre famille. Dawood a bu beaucoup d'alcool à bord et le personnel du navire lui reprochait son comportement agressif. Un soir, une violente dispute a éclaté. Effrayée, Elham s'est cachée dans la cabine de leurs amis. Dawood l'a retrouvée et a commencé à la frapper sous les yeux de leurs amis. Il lui a dit qu'il allait la tuer et la jeter par dessus bord. La dispute a continué dans la cabine d'Elham où Dawood a continué à la frapper et à déchirer ses vêtements. Elham a réussi à contacter la sécurité du navire qui lui a donné une autre cabine jusqu’à la fin de la croisière. Dawood est resté avec les enfants pendant les trois derniers jours et a tenté de les dresser contre leur mère.

$\mathrm{Au}$ retour de la croisière, Elham est allée vivre chez sa sœur dans la même ville. Elle retournait au domicile conjugal pour cuisiner, nettoyer et s'occuper des enfants pendant que Dawood était au travail. Avec l'aide de sa sœur, elle a contacté la police, mais a refusé de faire une déclaration. Deux mois plus tard, elle est retournée au domicile conjugal, mais elle a été reléguée au sous-sol.

Quelques semaines seulement après son retour, Dawood a menacé Elham avec le col d'une bouteille de bière cassée en exigeant qu'elle arrête de travailler. Elham a obtempéré. Une semaine plus tard, Elham a reproché à Dawood d'interroger les enfants et de fouiller dans ses affaires. Dawood a commencé à se moquer d'elle en la filmant avec un caméscope (ce qu'il avait déjà fait). Elham a essayé d’attraper le caméscope, mais Dawood l'a poussée au sol où elle est tombée sur les coudes en se cognant la tête et en se blessant au cou. Elle a dit à Dawood qu'elle ne voulait plus subir ces mauvais traitements. Elle a appelé la police, qui lui a demandé de déménager dans un endroit sûr. Les officiers ont occupé Dawood pendant qu'Elham faisait ses bagages. Elle est allée dans un refuge avec ses enfants. Aucune charge n'a été retenue contre Dawood qui a essayé de convaincre la police que sa femme l'avait attaqué. La police a contacté la Société d'aide à l'enfance et les enfants ont suivi des séances de counseling sur la maltraitance organisées dans le refuge. Elham a demandé une ordonnance d'éloignement contre son conjoint qui a été accordée pour une durée de seize mois. Se sentant en sécurité avec ses enfants, elle a décidé d’annuler la mesure d'interdiction pour minimiser les conflits au sein de la famille.

\section{Résolution}


À la fin de l'ordonnance d'éloignement, Elham et son mari ont convenu d'un calendrier de visites après intervention du tribunal. Un an plus tard, un nouvel incident a éclaté entre Dawood et ses enfants. Celui-ci a été interdit de communication pendant six (6) mois. La Société d’aide à l'enfance a été saisie. Elham souhaitait commencer une nouvelle vie et fournir de l'amour, de la stabilité et des soins à ses enfants. Dawood a continuéà essayer tromper les tribunaux en donnant une version différente des événements ayant conduit à la séparation. Le Bureau de l'avocat des enfants (Office of the Children's Lawyer, OCL) a été saisi pour représenter l'intérêt supérieur des enfants. Elham a demandé une garde conjointe et serait favorable à un droit de visite de son mari, si celui-ci demandait de l'aide pour régler ses problèmes de colère et d'alcool. Elle demande une pension alimentaire pour les enfants et l'épouse. 
15.

ÉTUDE DE CAS 14 : RITU ET SATINDER

\begin{tabular}{|l|l|l|}
\hline Profil & Femme & Homme \\
\hline Nom & Ritu & Satinder \\
\hline Âge au moment du mariage & 27 & 30 \\
\hline Âge* & 28 & 31 \\
\hline Pays d'origine & Inde & Inde \\
\hline Religion & Sikh & Sikh \\
\hline Éducation & Premier cycle universitaire & Premier cycle universitaire \\
\hline Connaissance de l'anglais & Bon niveau & Bon niveau \\
\hline Emploi avant la migration & Informatique & Aucune information disponible \\
\hline Emploi & $\begin{array}{l}\text { Travail dans une chaine de } \\
\text { restauration rapide }\end{array}$ & Responsable des ressources humaines \\
\hline Catégorie d'immigration & $\begin{array}{l}\text { Regroupement familial : Satinder a } \\
\text { parrainé Ritu dans le cadre du } \\
\text { programme de parrainage des } \\
\text { conjoints }\end{array}$ & Aucune information disponible \\
\hline Statut d'immigrant* & Résidente permanente (2016) & Citoyen \\
\hline & $\mathbf{1}$ & \\
\hline
\end{tabular}

* Au moment de la demande auprès de la Cour de la famille

\section{Parcours avant la migration}

Ritu a obtenu un baccalauréat en administration des affaires en Inde. Pendant ses études, elle a travaillé à temps plein dans une grande entreprise de conception Web et de marketing numérique en Inde. Elle a arrêté de travailler pendant une courte période pour préparer ses examens universitaires, mais elle a repris son travail 
après son mariage comme cadre supérieure chez un autre fournisseur de solution Web. En prévision de son déménagement au Canada, elle a quitté son poste au bout de cinq (5) mois pour préparer les examens du IELTS (Système international d'évaluation de la langue anglaise). Mais Satinder est venu en Inde en avril 2016 pour subir une chirurgie de la colonne vertébrale. Ritu n’a pas terminé ses examens IELTS, car elle a pris soin de Satinder pendant son rétablissement.

Ritu et Satinder se sont rencontrés sur un site internet de mariage destiné aux personnes de l’Asie du Sud. Ils ont commencé à se parler à distance et sont restés en contact permanent pendant l'année qui a précédé leur mariage. Au moment du mariage, Ritu avait vingt-sept ans et Satinder vingt-neuf. La famille de Satinder est venue en Inde pour lui donner leur «Shagun » (gage de bonne chance) selon la tradition indienne. Avant le mariage, Satinder a envoyé une note de remerciement au site de mariage pour exprimer sa gratitude. Ritu et Satinder se sont mariés en Inde. À la fin de la lune de miel, Satinder est retourné au Canada. Le couple se parlait quotidiennement au téléphone, parfois pendant quatre heures. Lors de ces conversations, Satinder a promis à Ritu qu'il l'aiderait à poursuivre ses études après son arrivée au Canada afin qu'elle puisse travailler dans la programmation de logiciels. Un an après leur mariage, Ritu est arrivée au Canada à titre de résidente permanente dans le cadre du programme de parrainage des conjoints.

\section{Installation au Canada}

Peu temps après l'arrivée de Ritu au Canada, Satinder lui a recommandé de terminer sa formation d'assistante dentaire afin d'assurer un revenu stable et de poursuivre ensuite sa carrière dans le développement de logiciels. Elle donc commencé des études d’assistante dentaire deux mois après son arrivée au Canada. Toutefois, au moment de demander un prêt étudiant, Satinder a refusé de cosigner le document et a déclaré que ce n'était pas la bonne solution. Quatre mois après son arrivée au Canada, Ritu a trouvé un travail à temps plein comme ouvrière dans une usine. Elle a été licenciée le mois suivant. Depuis lors, elle n’a pas été en mesure de subvenir à ses besoins financiers.

\section{Violence domestique}

Lorsque Ritu est arrivée au Canada en mai 2016, elle a emménagé chez la famille de Satinder. Quinze jours plus tard, Satinder l'a informé qu'il avait un partenaire sexuel masculin et qu'il avait besoin d'une femme pour dissimuler son orientation sexuelle. Les parents de Satinder étaient au courant de son orientation sexuelle avant le mariage avec Ritu. Selon Ritu, Satinder avait consommé le mariage pendant la lune de miel afin qu'elle ne se doute pas de son orientation sexuelle.

Satinder a suggéré à Ritu que le couple vive avec son partenaire masculin dans une nouvelle maison, ce que Ritu a refusé. Satinder lui a alors dit qu'elle devait continuer à vivre chez ses parents pendant qu'il emménageait avec son partenaire masculin.

Après son déménagement, Satinder a continué à rendre visite régulièrement à sa famille. Lors d'une visite, il a eu des relations sexuelles avec Ritu sans son consentement. Dès le début, Ritu a subi des violences verbales, physiques et sexuelles de la part de Satinder et de sa belle-famille. Ses beaux-parents craignaient que 
l'orientation sexuelle de Satinder ne porte atteinte à la réputation de la famille et souhaitaient que Ritu joue le rôle de l'épouse pour dissimuler la sexualité de Satinder. Les beaux-parents forçaient le couple à assister à des événements sociaux pour maintenir l'apparence d'un couple hétérosexuel heureux. Ils menaçaient Ritu d'expulsion et de ruiner sa réputation si elle ne se conformait pas. Angoissé et énervé par la situation, Satinder devenait de plus en plus violent avec Ritu.

Au début du séjour de Ritu au Canada, ses beaux-parents lui avaient pris ses bijoux en or pour les déposer dans un coffre à la banque. Ils contrôlaient également tous ses mouvements et l'interdisaient de sortir et de fréquenter des amis. Ritu refusait toujours de vivre avec Satinder et son partenaire masculin. Satinder a alors demandé le divorce à Ritu. Il a tenté à plusieurs reprises de forcer Ritu à signer les papiers du divorce. Un jour, alors qu'elle refusait, il l'a fait tomber par terre en lui claquant la porte au nez. Entre-temps, les parents de Satinder souhaitaient toujours que le mariage apparaisse comme une relation hétérosexuelle normale et faisaient pression sur Ritu pour qu'elle donne naissance à un enfant pour asseoir définitivement cette image.

En septembre 2016, quatre mois après son arrivée au Canada, la belle-famille a appelé les parents de Ritu pour leur annoncer que le mariage était rompu, car Ritu entretenait une liaison avec un autre homme. C'était un mensonge éhonté. Craignant pour sa sécurité, Ritu a appelé la police qui l'a conduit dans un refuge pour femmes. Elle a découvert sur place que Satinder avait installé une application de suivi sur son téléphone. Pendant le séjour de Ritu au refuge, Satinder a prétendu qu'elle avait créé un faux profil Instagram avec l'intention malveillante de lui causer du tort. Il a déclaré qu'elle publiait des photos de lui et de son partenaire. Ritu a nié ces allégations et a coopéré pleinement avec la police pendant toute l'enquête.

\section{Résolution}

Satinder a déposé une demande de divorce. Ritu a demandé une pension alimentaire et une part égale du réglement, ainsi que ses bijoux en or. Les revenus de Satinder au moment de la séparation ont été estimés à environ 60000 dollars par an. Elle a reçu une somme de 2000 \$ à titre de dernier règlement de la pension alimentaire pour époux, car Satinder avait quitté son emploi pour continuer ses études de baccalauréat et que sa seule source de revenus à l'époque était le RAFEO. Durant l'examen de son affaire par la Cour de la famille, Ritu a reçu une aide du programme Ontario au travail pour perfectionner ses compétences linguistiques et professionnelles. Ritu poursuit actuellement des études postsecondaires dans un collège communautaire. Elle est endettée à cause de ses prêts étudiants. 


\section{ÉTUDE DE CAS 15 : AYESHA ET KABIR}

\begin{tabular}{|l|l|l|}
\hline Profil & Femme & Homme \\
\hline Nom & Ayesha & Kabir \\
\hline Âge au moment du mariage & 25 & 27 \\
\hline Âge* & 31 & 33 \\
\hline Pays d'origine & Bangladesh & Bangladesh \\
\hline Religion & Musulman & Musulman \\
\hline Éducation & Premier cycle universitaire & Deuxième cycle universitaire \\
\hline Connaissance de l'anglais & Bon niveau & Bon niveau \\
\hline Emploi avant la migration & Étudiant & Étudiant \\
\hline Emploi & Sans emploi & Informatique \\
\hline Catégorie d'immigration & & $\begin{array}{l}\text { Catégorie du regroupement familial : } \\
\text { Ayesha a parrainé Kabir dans le cadre } \\
\text { du programme de parrainage des } \\
\text { conjoints }\end{array}$ \\
\hline $\begin{array}{l}\text { Nombre d'années de mariage* }: \mathbf{6} \\
\text { Enfants* }\end{array}$ & Aucune information disponible & Résident permanent (2005) \\
\hline
\end{tabular}

* Au moment de la demande auprès de la Cour de la famille

\section{Parcours avant la migration}

La famille biologique d'Ayesha est originaire du Bangladesh et a immigré au Canada. Sa famille pratiquait le soufisme, une forme spirituelle de l'islam. À l'époque de son mariage, Ayesha suivait des études universitaires. Elle était sur le point d'obtenir le baccalauréat et vivait depuis quatre ans avec sa famille à Toronto. Ayesha était une chanteuse bengali talentueuse et capable d'accompagner des chanteurs professionnels. Tous les membres 
de sa famille étaient citoyens canadiens. Kabir vivait au Royaume-Uni où il terminait ses études supérieures en technologie. Kabir venait d'une famille aisée, son père était un haut fonctionnaire du secteur du transport aérien. Le mariage avait été arrangé par les deux familles. La cérémonie du mariage a eu lieu au Bangladesh en 2004.

\section{Installation au Canada}

Ayesha a terminé ses études de baccalauréat en juin 2005, peu après le mariage. Elle a parrainé Kabir pour qu'il vienne au Canada. Il est arrivé un mois après l'obtention de son diplôme. Ayesha et Kabir ont commencé leur vie conjugale dans une grande ville canadienne. Kabir ne voulait pas que sa femme travaille après leur mariage. Kabir a trouvé un travail au service informatique d'une banque. Ayesha est tombée enceinte peu après leur mariage, et leur enfant est né en 2006.

\section{Violence domestique}

Les mauvais traitements ont commencé au tout début de leur vie commune. Kabir se plaignait de la cuisine d'Ayesha. Un jour, il a maintenu le visage d'Ayesha au-dessus d'une casserole d'huile bouillante en lui disant qu'il allait lui enseigner qu'elle était la bonne température de cuisson. Effrayée par ses accès de colère et sa violence, Ayesha s'est rapidement soumise à la volonté de son mari. Durant toute sa grossesse, Kabir lui donnait des coups de pied, la poussait et la frappait. Kabir ne voulait pas d'enfant et faisait pression sur Ayesha pour qu'elle avorte. Il a demandé l'aide de sa famille élargie pour tenter de la convaincre, mais celle-ci a toujours refusé de le faire.

Au quatrième mois de sa grossesse, Ayesh a révélé à son médecin de famille les problèmes de sa relation avec Kabir. Le médecin a aiguillé le couple vers un psychiatre qui a suggéré à Kabir de consulter un autre psychiatre pour régler ses problèmes de colère. Kabir a mal pris cette recommandation et a cessé d'aller voir le psychiatre. Il a interdit à Ayesha de se rendre à d'autres rendez-vous et il est devenu de plus en plus violent.

Leur fils est né par césarienne et les parents d’Ayesha sont venus aider la famille pendant une semaine. Cette visite a été écourtée quand Kabir a insulté la mère d'Ayesha. Ayesha a pu obtenir un certain soutien. L'infirmière de santé publique qui venait à domicile pour aider Ayesha après l'accouchement a remarqué que cette dernière présentait des troubles de santé mentale. Elle pensait qu'il s'agissait d'une dépression postnatale et apportait une aide émotionnelle à Ayesha. Ayesha devait accomplir toutes les tâches ménagères, élever l'enfant et servir la famille élargie de Kabir. Perturbé par les cris du nouveau-né, Kabir a demandé à Ayesha de dormir avec leur fils sur un canapé gigogne dans une autre pièce. Un an après la naissance du bébé, Ayesha a déménagé chez ses parents pendant plusieurs mois tandis que le beau-frère de Kabir emménageait au domicile conjugal.

Au fil des ans, Kabir imposait une discipline stricte toujours aussi stricte, exigeant de l'ordre et de l'obéissance de la part d'Ayesha et de leur fils. Si son fils ne se conformait pas immédiatement à ses ordres, Kabir le réprimandait, le secouait et le frappait. L'enfant avait peur de son père. Ayesha ne pouvait pas intervenir, car elle aurait été battue. Kabir a trouvé un nouvel emploi et la famille a déménagé dans une nouvelle maison à partir de juin 2010. Le titre de propriété de la maison était établi au seul nom de Kabir. Kabir gagnait beaucoup 
d'argent et a commencé à suivre des cours de troisième cycle universitaire dans une université locale. Kabir disait à Ayesha que son domaine était la maison qu'elle devait ranger et organiser, sous peine d'être battue.

Lors d'une visite en janvier 2010, l'oncle d'Ayesha a assisté aux mauvais traitements infligés par Kabir à Ayesha et à son fils. Kabir avait frappé Ayesha avec une chaussure et l'enfant était tellement indigné qu'il a essayé d'intervenir. Kabir est devenu furieux et a poussé l'enfant sur le canapé. Lorsque l'enfant est intervenu à nouveau, Kabir l'a enfermé dans les toilettes et a éteint la lumière. L'enfant était terrifié.

Les mauvais traitements ont continué et Ayesha a commencé à avoir des troubles psychologiques non traités. La violence s'est intensifiée à partir de 2007 pour atteindre un pic en 2010. Kabi disait à Ayesha qu'il l'aurait déjà tuée, si sa famille n’habitait pas au Canada. Il menaçait de la tuer et de se suicider ensuite. Il la menaçait avec un couteau et la traitait de femme «ignorante » et «stupide ». Son fils était témoin de la plupart de ces mauvais traitements et essayait de protéger sa mère.

En août 2010, Kabir a appelé les parents d'Ayesha pour leur demander de reprendre leur fille et de ne pas revenir tant qu'elle ne se serait pas « améliorée ». Ayesha a commencé à vivre chez ses parents avec son fils. Un jour, son père l'a emmenée chez le médecin de famille qui a aiguillé Ayesha vers une clinique de santé mentale. Elle a commencé un traitement en octobre 2010. La clinique a demandé au mari d'accompagner son épouse à une réunion d'admission avec un travailleur social. Kabir a répondu qu'il ne pouvait pas se rendre à l'hôpital et la clinique a contacté le père d'Ayesha pour lui demander de l'accompagner. Le lendemain, le père a pris rendezvous et Ayesh a rencontré un psychiatre. Au cours de la discussion, Ayesha a révélé qu'elle était terrifiée par son mari. Elle a déclaré au médecin qu'elle pensait que son mari avait des pouvoirs spéciaux et qu'elle avait peur de lui. Le psychiatre lui a dit qu'il était obligé de signaler ces faits à la police et à la Société d'aide à l'enfance et qu'il était préférable qu'elle le fasse elle-même. Ayesha a alors dénoncé les mauvais traitements à la police, qui l'a emmenée dans un refuge avec son enfant. Elle est restée pendant une nuit dans le refuge avant d'emménager dans la maison de ses parents. Kabir a été arrêté et accusé de voies de fait, d'agression avec une arme et de menaces. Il a été libéré avec interdiction de tout contact avec elle et son fils. La Société d'aide à l'enfance a été appelée au moment de l'intervention de la police et un employé s'est rendu au domicile des parents d'Ayesha pour évaluer les risques. L'objectif était de vérifier que l'enfant était en sécurité et d'offrir un soutien à Ayesha en cas de besoin.

\section{Résolution}

Kabir a entamé une procédure judiciaire pour obtenir le droit de garde et de visite de l'enfant. Ayesha a demandé la garde exclusive de son fils avec un droit de visite pour Kabir. La cour a ordonné une évaluation en vertu de l'article 30 de la Loi portant réforme du droit de l'enfance (LRO) et un psychologue a recommandé d'accorder la garde exclusive au père. Cette évaluation permet de protéger l'enfant et de répondre à ses besoins quand il existe un doute quant à la capacité de l'un des parents, notamment en ce qui concerne la santé mentale. Ayesha maitrise désormais ses problèmes de santé mentale et élève son enfant avec succès avec l'aide de ses parents. Pendant toute la durée de la procédure, le père a obtenu un droit de visite surveillé de l'enfant.

Ayesha a contesté l'évaluation en vertu de l'article 30, car elle ne tenait pas compte du problème des accès 
de colère de Kabir et des violences domestiques. Un rapport critique été réalisé : il recommande qu'Ayesha conserve la garde de son fils et qu'un Cercle de soutien et de responsabilité (CSR) soit formé autour d'elle pour s'assurer que les besoins de l'enfant soient satisfaits. L’affaire a été finalement réglée par le biais d'une entente de garde parallèle où Ayesha déciderait des questions de religion et de santé de l'enfant et le père prendrait des décisions en matière d'éducation de l'enfant. L'enfant réside principalement chez sa mère avec un droit de visite de son père. Ayesha reçoit une prestation alimentaire pour le conjoint et pour l'enfant. Le domicile conjugal a été vendu et Ayesha a reçu sa part de la vente. 
PARTIE II

QUESTIONS POUR ENGAGER UNE RÉFLEXION ET UNE DISCUSSION 



\section{VULNÉRABILITÉS INTERSECTIONNELLES}

1. Quels sont les facteurs qui peuvent contribuer à rendre les femmes immigrées et racialisées vulnérables à la violence?

2. Pourquoi est-il si important de comprendre les différents éléments du parcours d'une femme lorsque vous la défendez dans une affaire de violence domestique?

3. Quelles sont les conséquences de la violence domestique aux différents stades du développement des enfants?

4. Quels sont les différents effets des situations de maltraitance sur les individus, les familles et les communautés?

5. Quels sont les obstacles structurels auxquels se heurtent les femmes immigrées, racialisées et victimes de violence domestique?

6. Comment allez-vous travailler avec ces femmes pour leur faire surmonter ces obstacles et les conséquences de ces difficultés sur leur vie personnelle?

7. Quelles sont les institutions et les mesures administratives (immigration, protection de l'enfance, police, appareil judiciaire) auxquelles font face les femmes victimes de violence domestique ? Dans quelle mesure ces directives aident-elles à réduire ou à accroitre les vulnérabilités de ces femmes ? En tant qu'assistant(e) social(e), quelles pistes exploreriez-vous pour travailler avec ces femmes et les institutions de manière à négocier et à traiter les problèmes résultant de ces politiques ?

8. Dans le cadre de ces études de cas, comment analysez-vous les conflits entre les institutions et leurs politiques actuelles et le contexte culturel des femmes victimes de violence ? En tant qu'assistant(e) social(e), quelle serait votre position et comment travaillerez-vous avec ces femmes et les institutions pour surmonter les tensions et résoudre les problèmes?

9. Quel rôle joue la présence des enfants? Aide-t-elle ou enferme-t-elle davantage les femmes confrontées à la violence domestique ? Dans quelle mesure l'intervention des Sociétés d'aide à l'enfance facilite-t-elle ou complique-t-elle les choses pour la famille?

10. Dans certains cas, la famille de la femme insiste pour que celle-ci poursuive sa relation conjugale violente. Pourquoi ces familles insistent-elles pour que leurs filles continuent à vivre dans une telle situation de maltraitance ? Quelles sont les attentes en matière de sexe et de culture qui imposent ces conceptions et ces comportements?

11. Le traitement préférentiel accordé aux hommes est dominant dans de nombreuses cultures. Cela est mis en évidence dans les articles suivants : 
- Sex ratios at birth among second-generation mothers of South Asian ethnicity in Ontario, Canada: a retrospective population-based cohort study ${ }^{\circ}$ Preference for boys persists among 2 nd generation South Asian parents, study finds

12. Dans quelle mesure, ce traitement préférentiel influe-t-il sur les cas de violence domestique et les réponses apportées à ces cas? 
18.

\section{SUBJECTIVITÉ ET LOCALISATION SOCIALE DES PRATICIENS}

1. Dans quelle mesure, votre subjectivité et votre situation géographique influencent-elles votre point de vue sur la violence domestique subie par les femmes immigrées et racialisées. Plus précisément, quels préjugés et stéréotypes personnels, ces études de cas ont-elles fait apparaître à l'égard de ces femmes, des auteurs des violences et de la famille?

2. Dans quelle mesure votre subjectivité et votre position sociale affectent-elles votre travail avec ces femmes ? Comment aborderiez-vous cette question afin de construire une relation respectueuse et de travail avec ces femmes? 
19.

\section{POLITIQUE ET DROIT SOCIAUX}

1. Quelles sont les protections juridiques offertes aux victimes de violence domestique au Canada ? Quels types d'accords internationaux régissent ou influencent le traitement des cas de violence domestique lorsque la victime/l'auteur n'est pas un citoyen canadien ?

2. Dans quelle mesure, le système de la justice pénale aide-t-il et entrave-t-il les femmes confrontées à la violence domestique?

3. Quels sont les efforts de sensibilisation actuellement en cours pour prévenir la violence domestique et aider les victimes de violence domestique ? Comment améliorer ce travail de sensibilisation ?

4. Dans quelle mesure le manque de coordination entre la famille et le système de la justice pénale affecte-til les victimes de violence familiale et, en particulier, les enfants?

5. Quels pourraient être les avantages d'un triage précoce des affaires difficiles par les tribunaux de la famille et de l'affectation de ressources judiciaires supplémentaires à ces affaires pour améliorer l'accès à la justice des victimes de violence domestique?

6. À l'heure actuelle, chaque comparution devant la Cour supérieure de justice concernant une affaire de droit de la famille est traitée par un juge différent, qui ne connaît pas forcément tout l'historique de l'affaire. Pensez-vous qu'une approche basée sur une « gestion par cas » des affaires de violence domestique qui serait adoptée par tous les tribunaux de la famille (où le même juge superviserait l'avancement, programmerait et dirigerait les conférences et les requêtes dans les affaires de droit de la famille) serait une stratégie efficace, notamment dans les affaires de violence domestique? 
20.

\section{MIGRATION ET TRANSNATIONALISME}

1. Quel rôle les relations transnationales jouent-elles dans la vie des femmes immigrées?

2. De quelle manière, les relations transnationales représentent-elles un soutien ou un défi supplémentaire pour les femmes immigrées?

3. Dans quelle mesure, le fait de venir s'installer dans un nouveau pays contribue-t-il à l'isolement social et à la vulnérabilité de ces femmes dans chacune de ces études de cas ?

4. Quelles sont les particularités de la violence domestique subie par les femmes immigrées? 


\section{SOUTIEN AUX VICTIMES DE LA VIOLENCE DOMESTIQUE}

1. À quel type d'aide les femmes ayant participé à cette étude ont-elles eu accès ? Faites des recherches sur les aides auxquelles ces femmes pourraient avoir droit dans votre collectivité.

2. Quelles sont les meilleures pratiques pour aider les femmes immigrantes en situation de violence domestique ? Comment allez-vous travailler avec elles et à quels types de ressources pourriez-vous les adresser?

3. Comment allez-vous réagir dans une situation où une femme vous informe qu'elle est maltraitée et qu'elle souhaite rester avec son conjoint ? Quelles stratégies et ressources pouvez-vous conseiller à cette femme pour qu'elle puisse assurer sa sécurité tout en respectant son choix ?

4. Élaborez une stratégie d'intervention globale pour travailler avec les auteurs des actes de violence. Quelles ressources pensez-vous inclure?

5. Identifiez les problèmes rencontrés au niveau micro, mezzo et macro par chacune de ses femmes lorsqu'elles ont eu besoin d'aide. Quelles auraient pu être les interventions adéquates et culturellement appropriées à chaque niveau ?

6. Comment peut-on tirer parti de la communauté ? Comment peut-elle intervenir dans les cas de violence domestique?

7. À la lumière du cas présenté dans cette étude, comment le système aurait-il pu fonctionner plus efficacement pour soutenir la femme et sa famille? Que faudrait-il mettre en place pour changer ce type d'histoires et les expériences futures des femmes qui se trouveront dans une situation similaire?

8. Comment utiliseriez-vous une approche basée sur le traumatisme pour soutenir les femmes ayant subi des violences domestiques? 
22.

\section{RECHERCHE}

1. Si vous deviez interroger des femmes ayant subi des violences domestiques, quelles mesures prendriezvous pour minimiser les risques de préjudice pour les participants à votre recherche?

2. Quels pourraient être les concepts et la démarche d'une étude qui mettrait en avant les voix et les défis des femmes immigrantes, racialisées et victimes de violence domestique dans la région du Grand Toronto ? Comment aborderiez-vous les questions de pouvoir dans le processus de recherche? Comment utiliseriez-vous les résultats de cette recherche pour remettre en question/influencer le modèle existant des services offerts à ces femmes ? 